\section{BIOLOGICAL} CRYSTALLOGRAPHY

ISSN 1399-0047

\title{
Combination of X-ray crystallography, SAXS and DEER to obtain the structure of the FnIII-3,4 domains of integrin $\alpha 6 \beta 4$
}

\author{
Noelia Alonso-García, ${ }^{a}$ Inés García-Rubio, ${ }^{\mathrm{b}, \mathrm{c}}$ José A. Manso, ${ }^{\mathrm{a}}$ Rubén M. Buey, ${ }^{\mathrm{a}, \mathrm{d}}$ \\ Hector Urien, ${ }^{a}$ Arnoud Sonnenberg, ${ }^{\mathrm{e}}$ Gunnar Jeschke ${ }^{\mathrm{b}}$ and José M. de Pereda ${ }^{\mathrm{a} *}$
}

Received 19 November 2014

Accepted 5 February 2015

Edited by G. J. Kleywegt, EMBL-EBI, Hinxton, England

Keywords: integrin $\alpha 6 \beta 4$; Fnlll-3; Fnlll-4; smallangle $\mathrm{X}$-ray scattering; double electron-electron resonance.

PDB references: third FnIII domain of integrin $\beta 4,4 \mathrm{wtw}$; fourth FnIll domain of integrin $\beta 4$, $4 \mathrm{wtx}$

Supporting information: this article has supporting information at journals.iucr.org/d

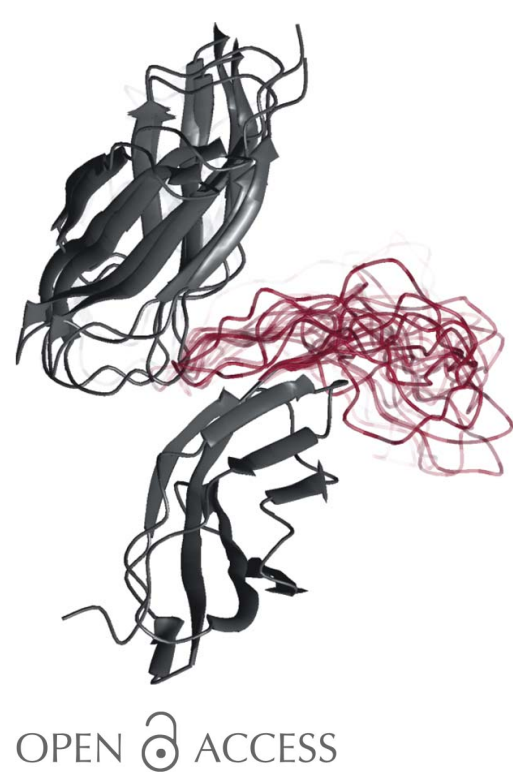

anstituto de Biología Molecular y Celular del Cancer, Consejo Superior de Investigaciones Científicas - University of Salamanca, Campus Unamuno, 37007 Salamanca, Spain, bLaboratory of Physical Chemistry, ETH Zürich, VladimirPrelog-Weg 2, CH-8093 Zürich, Switzerland, ' ${ }^{\mathrm{C}}$ Centro Universitario de la Defensa, Academia General Militar, Carretera de Huesca s/n, 50090 Zaragoza, Spain, ${ }^{d}$ Metabolic Engineering Group, Department of Microbiology and Genetics, University of Salamanca, Campus Unamuno, 37007 Salamanca, Spain, and ${ }^{\mathbf{e}}$ Netherlands Cancer Institute, Plesmanlaan 121,1066 CX Amsterdam, The Netherlands. *Correspondence e-mail: pereda@usal.es

Integrin $\alpha 6 \beta 4$ is a major component of hemidesmosomes that mediate the stable anchorage of epithelial cells to the underlying basement membrane. Integrin $\alpha 6 \beta 4$ has also been implicated in cell proliferation and migration and in carcinoma progression. The third and fourth fibronectin type III domains (FnIII-3,4) of integrin $\beta 4$ mediate binding to the hemidesmosomal proteins BPAG1e and BPAG2, and participate in signalling. Here, it is demonstrated that X-ray crystallography, small-angle X-ray scattering and double electronelectron resonance (DEER) complement each other to solve the structure of the FnIII-3,4 region. The crystal structures of the individual FnIII-3 and FnIII-4 domains were solved and the relative arrangement of the FnIII domains was elucidated by combining DEER with site-directed spin labelling. Multiple structures of the interdomain linker were modelled by Monte Carlo methods complying with DEER constraints, and the final structures were selected against experimental scattering data. FnIII-3,4 has a compact and cambered flat structure with an evolutionary conserved surface that is likely to correspond to a protein-interaction site. Finally, this hybrid method is of general application for the study of other macromolecules and complexes.

\section{Introduction}

Integrins are $\alpha \beta$ heterodimeric cell-surface adhesion and signalling receptors. In vertebrates, there are 24 receptors formed by combinations of $18 \alpha$ and eight $\beta$ subunits (Barczyk et al., 2010; Hynes, 2002). The $\alpha$ and $\beta$ subunits have large $\mathrm{N}$-terminal extracellular domains, a single transmembrane segment and C-terminal cytoplasmic tails that interact with cytoskeletal and signalling proteins (Campbell \& Humphries, 2011).

The $\beta 4$ subunit only pairs with the $\alpha 6$ subunit to form the $\alpha 6 \beta 4$ integrin, which is a laminin receptor required for maintaining the integrity of epithelia. Loss of $\alpha 6 \beta 4$ results in the blistering disease junctional epidermolysis bullosa associated with pyloric atresia (JEB-PA), which is characterized by fragility of the skin (Chung \& Uitto, 2010). $\alpha 6 \beta 4$ also participates in signalling pathways involved in keratinocyte proliferation and migration, and in carcinoma invasion and survival (Wilhelmsen et al., 2006).

$\alpha 6 \beta 4$ plays a major role in cell adhesion as an essential component of hemidesmosomes (HDs), junctional complexes that provide firm attachment of the basal layer of epithelial 
cells to the basement membrane by connecting the extracellular matrix to the intermediate filaments (de Pereda, Ortega et al., 2009; Margadant et al., 2008). In (pseudo) stratified epithelia the HDs contain $\alpha 6 \beta 4$, the bullous pemphigoid antigen 2 (BPAG2, also known as BP180 or collagen XVII), the tetraspanin CD151 and two members of the plakin family of cytolinkers: plectin and BPAG1e (BP230). $\alpha 6 \beta 4$ binds to CD151, BPAG2, plectin and BPAG1e, acting as a hub for the assembly, organization and regulation of HDs (Margadant $e t$ al., 2008).

The $\beta 4$ subunit mediates most of the intracellular interactions of $\alpha 6 \beta 4$. Mice carrying a deletion of the $\beta 4$ cytodomain had severe skin defects similar to those observed in the human disease JEB-PA and failed to form HDs (Murgia et al., 1998). The cytoplasmic moiety of $\beta 4$ ( $\sim 1000$ residues) is uniquely large among the integrin family. It has a modular structure that contains a Calx- $\beta$ domain (Alonso-García et al., 2009) followed by four fibronectin type III (FnIII) domains arranged in two pairs (FnIII-1,2 and FnIII-3,4; Fig. 1a). FnIII-2 and FnIII-3 are separated by a region of $\sim 140$ residues named the connecting segment (CS). Finally, an 86-residue C-terminal tail (C-tail) extends downstream of FnIII-4.

The FnIII domains of $\beta 4$ participate in protein-protein interactions in the HDs. FnIII-1,2 and part of the CS bind to the actin-binding domain of plectin (de Pereda, Ortega et al., 2009; Geerts et al., 1999). The C-terminal part of the CS, FnIII4 and the C-tail bind to the plakin domain of plectin (Frijns et al., 2012; Koster et al., 2004; Rezniczek et al., 1998). FnIII-3,4 together with the final sequence of the CS interact with BPAG1e, and FnIII-3 binds to BPAG2 (Koster et al., 2003).

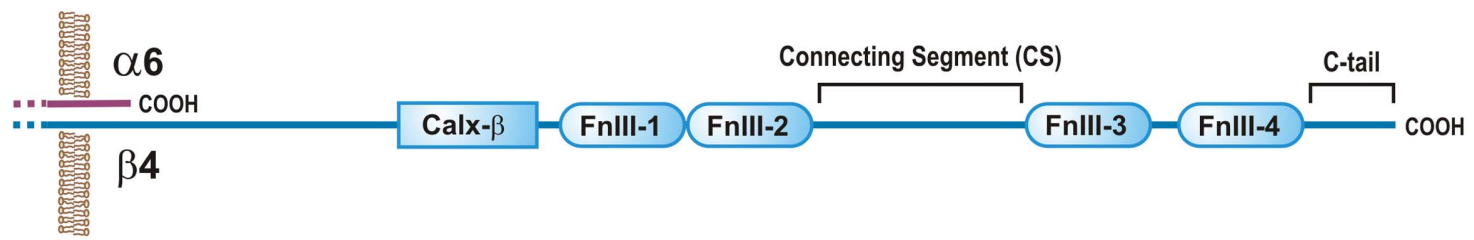

(a)
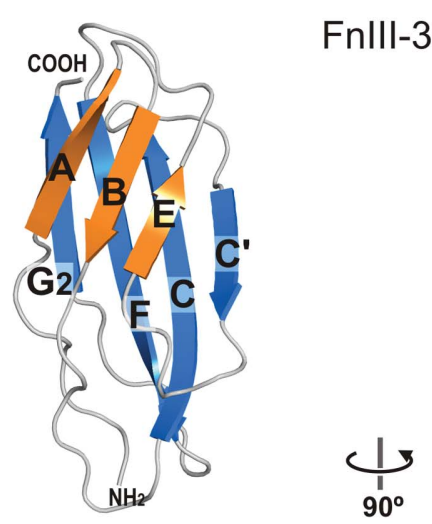

(b)

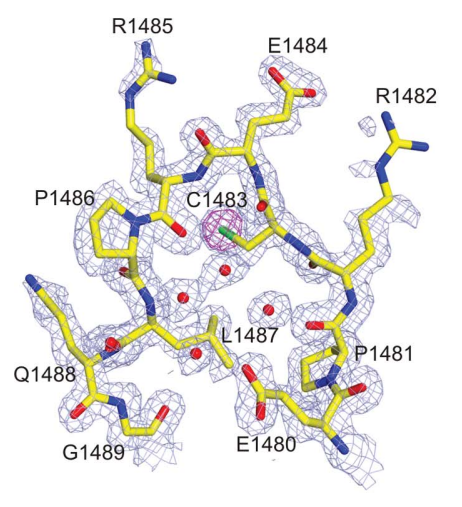

(d)
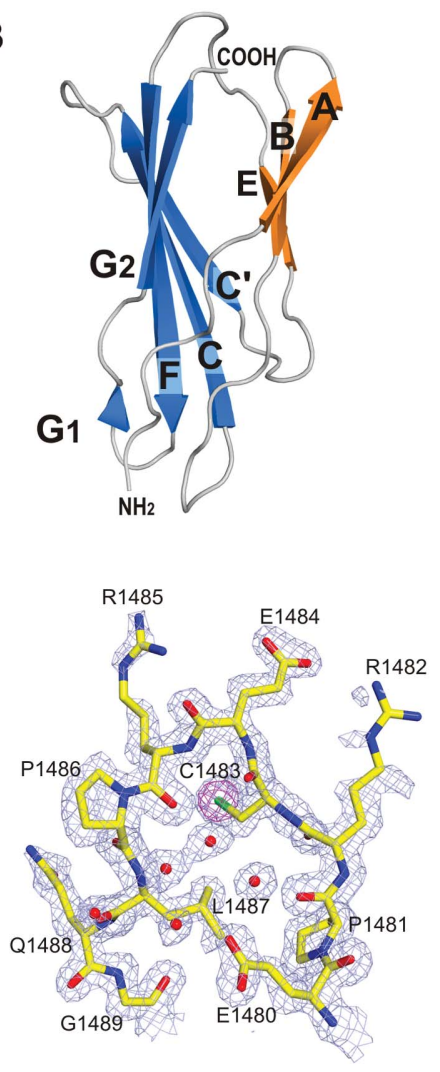
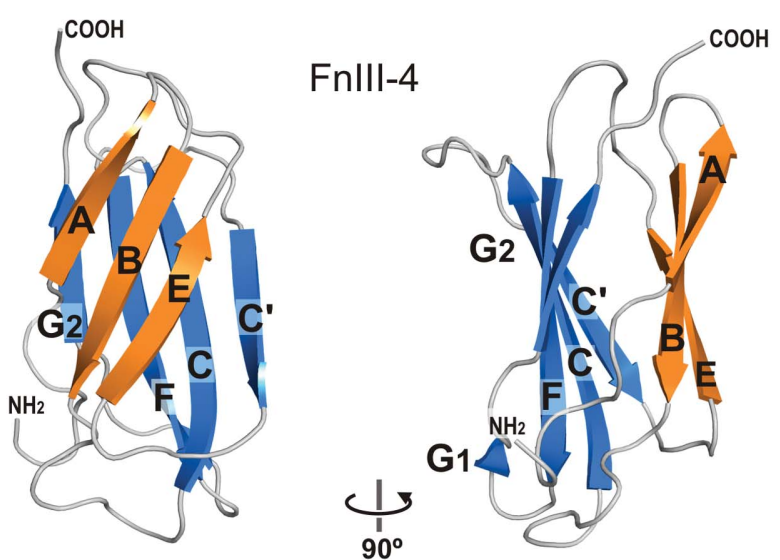

(c)

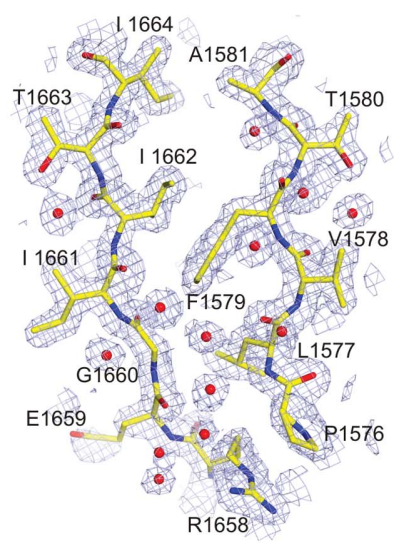

Figure 1

Crystal structures of the FnIII-3 and FnIII-4 domains of integrin $\beta 4$. (a) Diagram of the domain structure of the $\alpha 6 \beta 4$ cytoplasmic region. (b, $c$ ) Orthogonal views of ribbon representations of the crystal structures of the FnIII-3 $(b)$ and FnIII-4 $(c)$ domains. $(d, e)$ Stereoviews of $2 m F_{\text {obs }}-D F_{\text {calc }}$ simulated-annealing OMIT maps (contoured at $1 \sigma)$ of representative regions of FnIII-3 $(d)$ and FnIII-4 $(e)$ superimposed on the refined models. For FnIII-3, an anomalous difference map (contoured at $4 \sigma$ ) is shown in magenta. Phases were calculated from models in which the residues and the water molecules shown were removed and the $B$ factors were reset to the Wilson-plot value; the models were then refined by simulated annealing (start temperature $4000 \mathrm{~K}$ ). 
Table 1

Summary of crystallographic analysis.

Values in parentheses are for the outer resolution shell.

\begin{tabular}{|c|c|c|c|c|}
\hline \multirow{2}{*}{ Protein } & \multirow{2}{*}{$\frac{\text { FnIII-3 (1457-1548) }}{\text { Native }}$} & \multicolumn{3}{|c|}{ FnIII-4 (1572-1666) } \\
\hline & & Native 1 & EMTS soak & Native 2 \\
\hline \multicolumn{5}{|l|}{ Data collection } \\
\hline Space group & $I 2_{1} 2_{1} 2_{1}$ & $P 4_{1} 2_{1} 2$ & $P 4_{1} 2{ }_{1} 2$ & $P 4_{1} 2_{1} 2$ \\
\hline \multicolumn{5}{|l|}{ Unit-cell parameters $(\AA)$} \\
\hline$a$ & 66.7 & 56.9 & 56.9 & 57.3 \\
\hline$b$ & 68.3 & 56.9 & 56.9 & 57.3 \\
\hline$c$ & 88.4 & 78.4 & 78.4 & 76.3 \\
\hline Wavelength $(\AA)$ & 1.5418 & 1.5418 & 1.5418 & 0.9793 \\
\hline Resolution $(\AA)$ & $1.60(1.69-1.60)$ & $1.80(1.90-1.80)$ & $1.95(2.05-1.95)$ & $1.50(1.54-1.50)$ \\
\hline Unique reflections & 26721 & 11858 & $17812 \dagger$ & 19975 \\
\hline Average multiplicity & $9.0(8.1)$ & $19.7(20.1)$ & $15.0(14.6)$ & $24.3(26.3)$ \\
\hline Completeness $(\%)$ & $98.6(91.2)$ & $95.0(89.5)$ & $99.6(99.9)$ & $95.3(99.8)$ \\
\hline$R_{\text {meas }} \ddagger$ & $0.039(0.313)$ & $0.052(0.609)$ & $0.072(0.420)$ & $0.062(1.433)$ \\
\hline $\mathrm{CC}_{1 / 2}$ & $1.00(0.96)$ & $1.00(0.98)$ & $1.00(0.98)$ & $1.00(0.89)$ \\
\hline$\langle I / \sigma(I)\rangle$ & $41.7(7.7)$ & $45.1(7.8)$ & $32.4(8.8)$ & $33.9(3.3)$ \\
\hline \multicolumn{5}{|l|}{ SIRAS phasing } \\
\hline$R_{\text {iso }} \S$ & & & 0.344 & \\
\hline $\begin{array}{l}\text { Phasing power (iso, acentric/ } \\
\text { iso, centric/ano) }\end{array}$ & & & $2.38 / 2.06 / 2.36$ & \\
\hline FOM (acentric/centric) & & & $0.59 / 0.51$ & \\
\hline \multicolumn{5}{|l|}{ Model refinement } \\
\hline $\begin{array}{l}\text { No. of protein molecules } \\
\text { in asymmetric unit }\end{array}$ & 2 & & & 1 \\
\hline Resolution range $(\AA)$ & $35-1.60$ & & & $46-1.50$ \\
\hline Unique reflections (work/free) & $25369 / 1340$ & & & 18911/992 \\
\hline$R_{\text {work }} / R_{\text {free }} \uparrow$ & $0.151 / 0.180$ & & & $0.195 / 0.218$ \\
\hline \multicolumn{5}{|l|}{ No. of non-H atoms } \\
\hline Protein $\dagger \dagger$ & $826 / 822$ & & & 773 \\
\hline Waters & 222 & & & 122 \\
\hline Sulfates & 10 & & & - \\
\hline PEGs & 20 & & & - \\
\hline \multicolumn{5}{|l|}{ Average $B$ values $\left(\AA^{2}\right)$} \\
\hline Wilson plot & 14.2 & & & 20.0 \\
\hline Protein $\dagger \dagger$ & 19.2/20.9 & & & 28.5 \\
\hline Waters/sulfates/PEGs & 30.2 & & & 34.8 \\
\hline Sulfates & 31.3 & & & - \\
\hline PEGs & 27.6 & & & - \\
\hline \multicolumn{5}{|l|}{ R.m.s.d. from ideal geometry $\neq \ddagger$} \\
\hline Bond lengths $(\AA)$ & 0.009 & & & 0.012 \\
\hline Angles $\left({ }^{\circ}\right)$ & 1.2 & & & 1.3 \\
\hline \multicolumn{5}{|l|}{ R.m.s.d. $B$ factors $\S \S\left(\AA^{2}\right)$} \\
\hline Main chain & 1.5 & & & 3.9 \\
\hline Side chain & 3.4 & & & 6.2 \\
\hline \multicolumn{5}{|l|}{ Ramachandran plot $\uparrow$} \\
\hline Residues in favoured regions & $234[98.3]$ & & & $108[98.2 \%]$ \\
\hline Additionally allowed & $4[1.7 \%]$ & & & $2[1.8 \%]$ \\
\hline Outliers & $0[0.0 \%]$ & & & $0[0.0 \%]$ \\
\hline Side-chain rotamer outliers & $1[0.5 \%]$ & & & $1[1.2 \%]$ \\
\hline Clashscore & 0.60 & & & 0.66 \\
\hline PDB code & 4wtw & & & $4 \mathrm{wtx}$ \\
\hline
\end{tabular}

$\dagger$ Keeping Bijvoet pairs separate. $¥$ The redundancy-independent $R$ factor $R_{\text {meas }}=\sum_{h k l}\{N(h k l) /[N(h k l)-1]\}^{1 / 2}$ $\sum_{i}\left|I_{i}(h k l)-\langle I(h k l)\rangle\right| / \sum_{h k l} \sum_{i} I_{i}(h k l)$, where $I_{i}(h k l)$ is the $i$ th measurement and $\langle I(h k l)\rangle$ is the mean of all measurements of $I(h k l)$ (Diederichs \& Karplus, 1997). $\& R_{\text {iso }}=\sum_{h k l}|| F_{\text {der }}|-| F_{\text {nat }}|| / \sum_{h k l}\left|F_{\text {nat }}\right|$, where $F_{\text {der }}$ is the heavy-atom-derivative structure factor and $F_{\text {nat }}$ is the protein structure factor. $\uparrow R_{\text {work }}=\sum_{h k l}|| F_{\text {obs }}|-| F_{\text {calc }}|| / \sum_{h k l}\left|F_{\text {obs }}\right| ; R_{\text {free }}$ was calculated using a randomly chosen $5 \%$ of reflections that were not included in the refinement and $R_{\text {work }}$ was calculated for the remaining reflections. †† Values for each protein chain. $\$$ Referred to the ideal geometry defined by Engh \& Huber (1991). $\S \S$ Calculated for bonded atom pairs with MOLEMAN2 (Kleywegt, 1997). ศศ Values were obtained using MolProbity (Chen et al., 2010).

FnIII-3 has also been implicated in cellular signalling. Y1494 is required for the activation of phosphatidylinositol 3-kinase and stimulation of invasion after ligation of $\alpha 6 \beta 4$ (Shaw, 2001). Phosphorylation of Y1494, together with Y1257 in FnIII-2 and Y1440 in the CS, results in binding of the phosphatase Shp2 to $\beta 4$ and stimulation of the Erk pathway (Bertotti et al., 2006). $\alpha 6 \beta 4$ is also coupled via Shc to Erk through phosphorylation of Y1526 in FnIII-3 (Dans et al., 2001).

Yeast two-hybrid and blotoverlay experiments using $\beta 4$ fragments suggest that the CS and the C-tail interact with each other (Koster et al., 2004; Rezniczek et al., 1998). Recently, it has been shown that the $\mathrm{C}$-tail is in close proximity to the CS of the same molecule in keratinocytes, suggesting that $\beta 4$ adopts a folded-back structure in which the linker between FnIII-3 and FnIII-4 might allow a bent conformation (Frijns et al., 2012).

In spite of the role of FnIII-3,4 in protein-protein interactions and in the arrangement of the $\beta 4$ cytodomain, the structural organization of this region remained largely uncharacterized. Here, we have combined X-ray crystallography, small-angle X-ray scattering (SAXS) and electron paramagnetic resonance (EPR) spectroscopy to elucidate the structure of FnIII-3,4.

\section{Materials and methods}

\subsection{Protein expression and purification}

The cDNA sequences of human integrin $\beta 4$ (UniProt P16144-2) coding for the regions 1457-1548, 1572-1666 and 14571666 were amplified by polymerase chain reaction using IMAGE clone 3640058 (GenBank BE737196) as a template. The amplified DNA fragments were cloned into a modified version of the pET-15b vector (Alonso-García et al., 2009) using NdeI and BamHI sites that were introduced into the forward and reverse primers, respectively. Soluble proteins were produced in Escherichia coli strain BL21(DE3) grown in Terrific Broth medium supplemented with $100 \mathrm{mg} \mathrm{l}^{-1}$ ampicillin. Protein synthesis was induced with $0.2 \mathrm{~m} M$ isopropyl $\beta$-D-1-thiogalactopyranoside; the fragments 1457-1548 and 1457-1666 were expressed at $310 \mathrm{~K}$ for $3 \mathrm{~h}$, while the fragment $1572-1666$ was expressed 
at $288 \mathrm{~K}$ for $12 \mathrm{~h}$. The proteins were purified by nickel-affinity chromatography as described in García-Alvarez et al. (2003). The His tag was cleaved by digestion with Tobacco etch virus protease, which leaves four additional residues (sequence GSHM) coded by the vector at the N-terminus. The His tag was removed by a second nickel-affinity chromatography step. Finally, the proteins were dialyzed against the desired buffer and were concentrated by ultrafiltration using Amicon cells (Merck Millipore).

\subsection{Crystallization and structure determination of FnIII-3}

Crystals of FnIII-3 (residues 1457-1548) were grown by vapour diffusion at $277 \mathrm{~K}$ by mixing protein solution at $20 \mathrm{mg} \mathrm{ml}^{-1}$ in $20 \mathrm{~m} M$ Tris- $\mathrm{HCl} \mathrm{pH}$ 7.5, $4 \mathrm{mM}$ DTT with an equal volume of mother liquor consisting of $0.1 \mathrm{M}$ Tris- $\mathrm{HCl}$ pH 7.0, 12.5\% glycerol, 36\% PEG 600, $0.5 M$ ammonium sulfate. Crystals were cooled by direct immersion into liquid $\mathrm{N}_{2}$. Data were collected at $120 \mathrm{~K}$ using an FR591 rotatinganode generator (Bruker AXS) and a MAR345 detector (MAR Research). Diffraction intensities were indexed and integrated with $X D S$, reduced with $X S C A L E$ and converted into structure-factor moduli with XDSCONV (Kabsch, 2010).

The FnIII-3 crystal belonged to space group $I 2_{1} 2_{1} 2_{1}$ and contained two molecules in the asymmetric unit $(\sim 47 \%$ solvent content, Matthews coefficient $2.33 \AA^{3} \mathrm{Da}^{-1}$; Table 1). The structure was phased by molecular replacement using Phaser (McCoy et al., 2007) within the CCP4 suite (Winn et al., 2011). The structure of FnIII-2 from $\beta 4$ (PDB entry 1qg3; de Pereda et al.,1999) was used to build a mixed search model for molecular replacement by homology modelling with SCWRL 4 (Krivov et al., 2009). Refinement was performed against data to $1.60 \AA$ resolution using phenix.refine (Afonine et al., 2012) combined with manual model building using Coot (Emsley et al. 2010). Simulated annealing was used in the initial stages of refinement. Later on, restrained positional refinement, individual isotropic $B$-factor restrained refinement, bulk-solvent correction and refinement of translation/libration/screw (TLS) parameters were used. Two TLS groups, one for each protein molecule, were refined. $\mathrm{H}$ atoms were included using a riding model. Two electron densities with a tetrahedral shape whose centres corresponded to peaks in native anomalous difference maps were modelled as sulfate ions. The final model contains residues 1457-1548 in each protein chain; molecules $A$ and $B$ contain three (SHM) and one (M) additional residues at the $\mathrm{N}$-terminus, respectively, encoded by the vector. The structure also contains 219 solvent molecules and two PEG fragments. The model has excellent geometry, with 98.3 and $1.7 \%$ of the main-chain torsion angles located in the favoured and the additionally allowed regions, respectively, of the Ramachandran plot determined with MolProbity (Chen et al., 2010).

\subsection{Crystallization and structure determination of FnIII-4}

Crystals of FnIII-4 (residues 1572-1666) were obtained at $277 \mathrm{~K}$ by vapour diffusion; protein solution at $27 \mathrm{mg} \mathrm{ml}^{-1}$ in $10 \mathrm{~m} M$ Tris-HCl pH 7.5, $2 \mathrm{~m} M$ DTT was mixed with an equal volume of crystallization solution consisting of $0.1 M$ sodium acetate $\mathrm{pH}$ 4.2, $0.72 M \mathrm{NaH}_{2} \mathrm{PO}_{4}, 1.08 M \mathrm{~K}_{2} \mathrm{HPO}_{4}, 2 \mathrm{~m} M$ DTT. Before data collection, native crystals were transferred into a cryoprotectant solution consisting of $0.1 \mathrm{M}$ sodium acetate $\mathrm{pH}$ 4.2, $0.72 M \mathrm{NaH}_{2} \mathrm{PO}_{4}, 1.08 M \mathrm{~K}_{2} \mathrm{HPO}_{4}, 2 \mathrm{~m} M$ DTT, $5 \%$ glycerol and were cooled by immersion in liquid $\mathrm{N}_{2}$. Alternatively, a crystal was derivatized with heavy atoms by soaking it for $6 \mathrm{~h}$ at $277 \mathrm{~K}$ in $0.1 M$ sodium acetate $\mathrm{pH} 4.2$, $0.72 M \mathrm{NaH}_{2} \mathrm{PO}_{4}, 1.08 \mathrm{M} \mathrm{K}_{2} \mathrm{HPO}_{4}, 1 \mathrm{~m} M$ ethylmercurithiosalicylate (EMTS). Excess EMTS was removed by a brief transfer into the cryoprotectant solution without DTT prior to immersion in liquid $\mathrm{N}_{2}$. Data from initial native (native 1) and EMTS-derivatized crystals were collected at $100 \mathrm{~K}$ using a MICROSTAR-H rotating-anode generator (Bruker AXS). Subsequently, data from a second native crystal (native 2) were collected at $100 \mathrm{~K}$ on the PXIII beamline of the Swiss Light Source, Villigen, Switzerland. Diffraction data were processed as for the FnIII-3 crystals.

The crystals belonged to space group $P 4_{1} 2_{1} 2$ and contained a single FnIII-4 molecule in the asymmetric unit $(\sim 59 \%$ solvent content, Matthews coefficient $2.95 \AA^{3} \mathrm{Da}^{-1}$; Table 1). Phases were obtained by single isomorphous replacement with anomalous scattering (SIRAS) using the native 1 and mercury-derivative data sets. Determination of the heavyatom substructure (one high-occupancy $\mathrm{Hg}$ site and three minor sites), calculation of its approximate substructure amplitudes and initial phase calculations were performed with SHELXC/D/E (Sheldrick, 2010) and HKL2MAP (Pape \& Schneider, 2004). Phase probability distributions for the major $\mathrm{Hg}$ site were further refined with autoSHARP (Vonrhein et al., 2007), which allowed the confirmation of three minor $\mathrm{Hg}$ sites. The phases were improved and extended with SOLOMON (Abrahams \& Leslie, 1996). A map of outstanding quality and detail was calculated using the SIRAS-derived phases (Supplementary Fig. S1), which allowed the building of 94 out of 95 residues using $A R P / w A R P$ (Langer et al., 2008). Refinement was performed similarly as for the FnIII-3 structure. After initial refinement against the native 1 data to $1.80 \AA$ resolution, the structure was refined against the native 2 data set extending to $1.50 \AA$ resolution. The same subset of reflections, in the resolution range common to the two data sets, was used for the calculation of $R_{\text {free. }}$ The TLS parameters of five groups were refined. The refinement converged at $R_{\text {work }}$ and $R_{\text {free }}$ values of 0.195 and 0.218 , respectively. The refined model includes residues 1572-1666, two additional residues (HM) encoded by the vector at the $\mathrm{N}$-terminus and 122 solvent molecules. The structure has very good geometry; $98.2 \%$ of the main-chain torsion angles are in the most favoured regions of the Ramachandran plot and the remainder (1.8\%) fall into additionally allowed regions.

\subsection{Analysis of atomic structures}

Pairwise superimposition of structures was performed with LSQKAB (Kabsch, 1976). Simultaneous superimposition of multiple structures was performed with THESEUS, which applies a maximum-likelihood method (Theobald \& Steindel, 2012). Electrostatic properties were calculated with $A P B S$ 
(Baker et al., 2001) using the PARSE force field (Sitkoff et al., 1994), and the electrostatic potentials were compared with the webPIPSA server (Richter et al., 2008). Evolutionary conservation scores were calculated with ConSurf (Ashkenazy et al., 2010). Molecular figures were created using PyMOL (v.1.5.0.2; Schrödinger).

\subsection{SAXS measurements and analysis}

SAXS data were collected at the European Molecular Biology Laboratory (EMBL) on beamline P12 at Deutsches Elektronen Synchrotron (DESY), Hamburg, Germany using a Pilatus 2M detector (Dectris) with radiation of wavelength $1.24 \AA$ A. Proteins for SAXS analysis were additionally purified and equilibrated in $20 \mathrm{~m} M$ sodium phosphate $\mathrm{pH} 7.5,150 \mathrm{mM}$ $\mathrm{NaCl}, 5 \%$ glycerol, $3 \mathrm{mM}$ DTT by size-exclusion chromatography using a HiPrep Sephacryl S300 26/60 column (GE Healthcare). Data for wild-type FnIII-3,4 were collected at a sample-to-detector distance of $4.1 \mathrm{~m}$ over a scattering-vector range from 0.01 to $0.35 \AA^{-1}[q=(4 \pi \sin \theta) / \lambda$, where $2 \theta$ is the scattering angle]. Data for protein samples at 1.5, 3.0, 6.1, 12.2, 24.3 and $48.6 \mathrm{mg} \mathrm{ml}^{-1}$ and buffer were measured at $283 \mathrm{~K}$. No radiation damage was observed by comparison of 20 successive $0.05 \mathrm{~s}$ exposures. Data were processed and analyzed using the ATSAS package (Petoukhov et al., 2012). SAXS data for the FnIII-3,4 MTSL-labelled mutants were measured and processed similarly using a detector distance of $3.1 \mathrm{~m}(0.01<q$ $<0.44 \AA^{-1}$ ). The ensemble-optimization method (EOM) was used to analyze interdomain flexibility (Bernadó et al., 2007).

$A b$ initio shape reconstructions were calculated with DAMMIF (Franke \& Svergun, 2009) and DALAI_GA (Chacón et al., 2000). 20 independent models were generated with each program. Each set of structures were superimposed and an averaged model that represents the most populated volume within each set was calculated with DAMAVER (Volkov \& Svergun, 2003). For representation, volumetric maps were calculated from the bead models with the SITUS package (Wriggers, 2010).

\subsection{Site-directed spin labelling (SDSL)}

Cys substitutions, replacing Cys in the wild-type sequence or introducing new Cys residues, were created in the construct of the FnIII-3,4 fragment (1457-1666) by site-directed mutagenesis using the QuikChange method (Stratagene). The mutants were expressed at $288 \mathrm{~K}$, were purified as for the wild-type protein and were labelled with the thiol-reactive paramagnetic nitroxide probe $S$-(1-oxyl-2,2,5,5-tetramethyl2,5-dihydro-1 $H$-pyrrol-3-yl)methyl methanesulfonothioate (MTSL). Proteins at $200 \mu M$ in $20 \mathrm{~m} M$ sodium phosphate $\mathrm{pH}$ $7.5,150 \mathrm{~m} M \mathrm{NaCl}$ were incubated with a tenfold molar excess of MTSL for $1 \mathrm{~h}$ at room temperature and subsequently for $12 \mathrm{~h}$ at $277 \mathrm{~K}$. Excess free MTSL was removed by extensive dialysis against the same buffer. Alternatively, for SAXS analysis of MTSL-labelled samples, excess free MTSL was removed and the proteins were equilibrated in $20 \mathrm{~m} M$ sodium phosphate $\mathrm{pH} 7.5,150 \mathrm{mM} \mathrm{NaCl}, 5 \%$ glycerol by sizeexclusion chromatography using a Superdex 200 10/300 GL column (GE Healthcare). The total free thiol groups before and after labelling were determined by titration with 5,5'dithio-bis(2-nitrobenzoic acid) under denaturing conditions (García-Alvarez et al., 2003) and revealed complete labelling.

\subsection{Double electron-electron resonance (DEER) measurements and data analysis}

Solutions of MTSL-labelled proteins at a concentration between 100 and $200 \mu M$ were mixed in a 1:1 volume ratio with deuterated glycerol, transferred into EPR quartz tubes and stored in liquid $\mathrm{N}_{2}$ until measurement.

Q-band DEER measurements were performed using a homemade spectrometer (Gromov et al., 2001) working at a microwave frequency (mw) of $\sim 34.4 \mathrm{GHz}$ and equipped with a $150 \mathrm{~W}$ travelling-wave tube (Applied Systems Engineering Inc). A homemade probe head that allows the measurement of up to $3 \mathrm{~mm}$ sample tubes (Tschaggelar et al., 2009) was used. All experiments were performed at a temperature of $50 \mathrm{~K}$ using a continuous gas-flow cryostat refrigerated with $\mathrm{He}$ (Oxford Instruments).

The pulse sequence $(\pi / 2) \mathrm{mw}_{1}-\tau_{1}-(\pi) \mathrm{mw}_{1}-\tau_{1}-(\pi) \mathrm{mw}_{2}-\tau_{2}-$ $(\pi) \mathrm{mw}_{1}-\tau_{2}-$ echo was set with $\mathrm{mw}$ pulse lengths of $12 \mathrm{~ns}$ for all pulses, and deuterium nuclear modulations were averaged out by adding eight experiments with $\tau_{1}$ increments of $16 \mathrm{~ns}$. The pump mw frequency $\mathrm{mw}_{1}$ was set to the maximum absorption of the spectrum and the observer mw frequency $\mathrm{mw}_{2}$ was $80 \mathrm{MHz}$ lower. The length of the experimental traces was between 3 and $6 \mu$ s depending on the transverse relaxation times. The time points were collected with a repetition rate of $300 \mathrm{~Hz}$ and the dipolar evolution traces were accumulated for several hours.

DEER traces were analyzed using DeerAnalysis (Jeschke et al., 2006), which is available online at http://www.epr.ethz.ch/ software/index. An exponential background decay function was fitted to the final part of the traces and was subtracted from the experimental trace. From the resulting dipolar timeevolution trace, the distance distribution was determined by Tikhonov regularization. A numerical value for the average distance between probes was then obtained for every pair of spin labels $i\left(\delta_{i, \mathrm{EXP}}\right)$

Prediction of the populations of the conformations of the spin label attached to a certain position was performed from the structure of the individual domains using a library developed to model all possible conformations with a reduced number of significant rotamers (Polyhach et al., 2011). This approach is implemented in the open-source package $M M M$ (Multiscale Modeling of Macromolecules), a Matlab-based collection of routines accessible through a graphical user interface. Simulations of DEER experiments based on the protein structure and rotamer libraries were performed using the DEER window in $M M M$.

\subsection{Molecular modelling}

To determine the relative arrangement of two solids, one needs to determine six relative coordinates: three for the translation degrees of freedom and three for the rotational 
degrees of freedom. To test the possible structural models against the experimental data, a complete set of relative arrangements was generated using a homemade Matlab-based program. The models were produced by combining a threedimensional translational grid of $2 \AA$ step size with a threedimensional rotational grid containing pairs of angles $\alpha$ and $\beta$ defining points uniformly distributed over a sphere (with every such orientation covering a solid angle of $0.0385 \mathrm{sr}$ ) plus an angle $\gamma$ that was sampled every $10^{\circ}$. For every relative arrangement, the average position of the spin probe delivered by $M M M$ was recalculated and the distances between average positions of the different spin pairs were computed $\left(\delta_{i, \mathrm{MODEL}}\right)$. In order to rank the models according to the degree of agreement with the experimental data, the parameter $\sigma_{\text {DEER }}$, defined as follows, was calculated for every structural model,

$$
\sigma_{\mathrm{DEER}}=\left[\frac{1}{N} \sum_{i=1}^{N}\left(\delta_{i, \mathrm{DEER}}-\delta_{i, \mathrm{MODEL}}\right)^{2}\right]^{1 / 2}
$$

where $N$ is equal to the number of interdomain distances that we have used for determination of the relative arrangements of the two FnIII domains.

Coordinates of the linker that connects FnIII-3 and FnIII-4 were constructed by a Monte Carlo approach that takes into account standard bond lengths and angles, a statistical distribution of backbone dihedral angles that conforms to residue-specific Ramachandran plots derived from the whole PDB (Hovmöller et al., 2002) and the location by two DEER distance constraints of the spin label attached to C1559, a natural cysteine located in the central region of the linker. For every model of interdomain arrangement, backbone $\mathrm{N}, \mathrm{C}^{\alpha}$ and carbonyl $\mathrm{C}$ coordinates were computed from the backbone dihedrals using the SugetaMiyazawa algorithm (Sugeta \& Miyazawa, 1967) and assuming standard peptide-bond lengths and angles. Carbonyl $\mathrm{O}$ atoms were added in the peptide plane. The initial rotation matrix was computed according to Shimanouchi \& Mizushima (1955) from the backbone coordinates of the C-terminal residue of FnIII-3.

Linker models were rejected if they featured self-clashes or clashes with atoms in the FnIII-3 or FnIII-4 domains. Clashes were defined as an approach of two heavy atoms of nonconsecutive residues of closer than $2.5 \AA$.

Spin-label coordinates at residue C1559 were predicted by transforming the mean $\mathrm{N}-\mathrm{O}$ midpoint coordinate in

Figure 2 a rotamer library of an unrestricted MTSL side chain (Polyhach et al., 2011) from the peptide standard frame to the local residue frame. A new $\sigma_{\text {DEER }}$ parameter was calculated taking into account the interdomain distance constraints and also those obtained from measurements involving the linker cysteine $\mathrm{C} 1559$. The first 11 residues up to $\mathrm{C} 1559$ were modelled by the unrestricted Monte Carlo approach, whereas the remaining residues up to $\mathbf{S 1 5 7 2}$ were modelled by a Monte Carlo Metropolis approach to steer the linker towards the N-terminal residue of domain FnIII-4, with the target coordinate being the $\mathrm{C}^{\alpha}$ coordinate of this residue. Monte Carlo moves were accepted only if they moved the residue by at least $1.325 \AA$ towards the target coordinate. This value led to an acceptable success rate in linker-model generation, with success being defined by an approach of the modelled S1572 $\mathrm{C}^{\alpha}$ coordinate to the target coordinate of within $5 \AA$. The remaining difference was eliminated by evenly distributing it over all linker backbone atoms. Side groups were then added with SCWRL4 (Krivov et al., 2009). The scattering curves of the atomic models were calculated and compared with the experimental SAXS profile with CRYSOL (Svergun et al.,

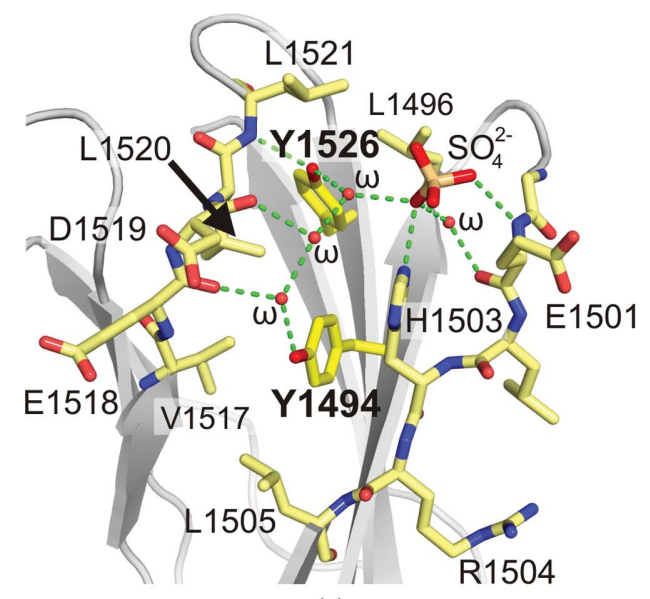

(a)

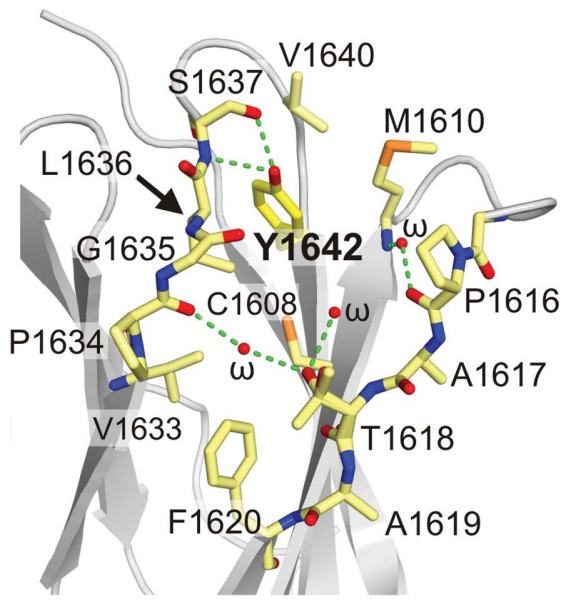

(c)

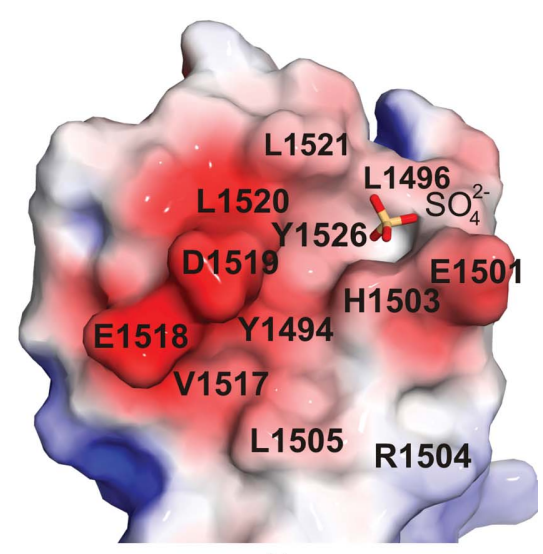

(b)

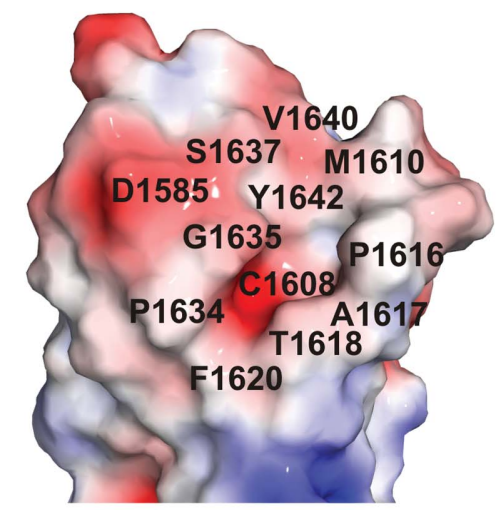

(d)

Structural environment of Tyr residues in FnIII-3 and FnIII-4. $(a, b)$ Close-up of FnIII-3 around Y1494 and Y1526 in stick $(a)$ and surface $(b)$ representation coloured by the electrostatic potential on the surface from $-3 k T / \mathrm{e}$ (red) to $3 k T / \mathrm{e}$ (blue). $(c, d)$ Close-up of Y1642 in the FnIII-4 in stick $(c)$ and surface $(d)$ representation coloured by electrostatic potential as in $(b)$. 
1995). Pair-distance distribution functions were calculated from atomic models with HYDROPRO (Ortega et al., 2011).

\subsection{Accession numbers}

The Protein Data Bank accession codes for the coordinates and structure factors of the FnIII-3 and FnIII-4 structures are 4wtw and 4wtx. SAXS data and models of wild-type FnIII-3,4 have been deposited in the Small Angle Scattering Biological Data Bank (SASBDB; Valentini et al., 2014) under code SASDAT6.

\section{Results}

\subsection{Crystal structures of FnIII-3 and FnIII-4}

The individual structures of the FnIII-3 and FnIII-4 domains of $\beta 4$ integrin were solved by X-ray crystallography. The structure of FnIII-3 (1457-1548) was refined against data to $1.60 \AA$ resolution. The asymmetric unit contains two copies

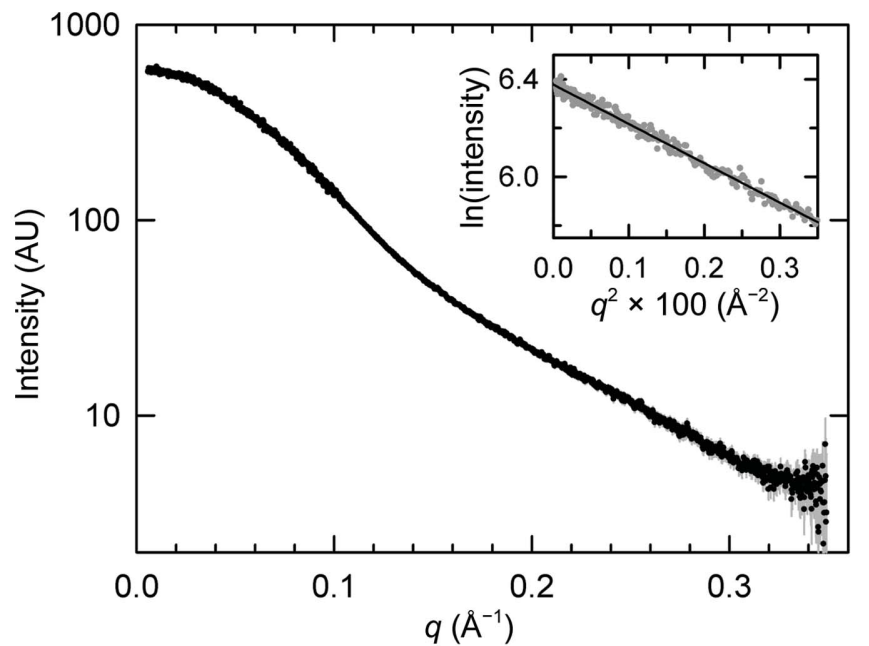

(a)

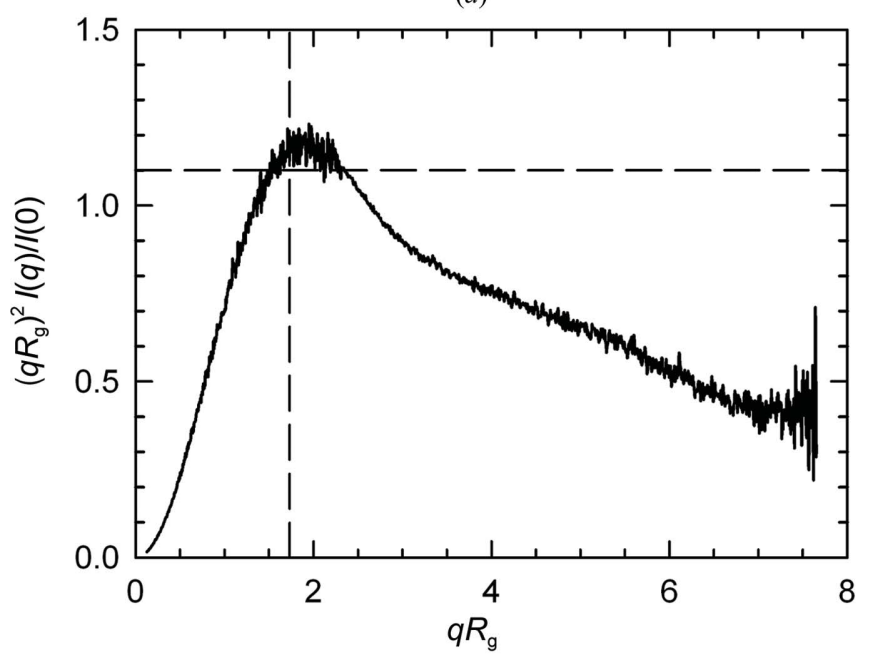

(c) of FnIII-3 that are almost identical. After superimposition, the root-mean-square difference (r.m.s.d.) for all main-chain atoms between the two molecules is $0.59 \AA$. The structure of the FnIII-4 domain (1572-1666) was refined against data extending to $1.50 \AA$ resolution; the FnIII-4 crystals contain a single protein molecule in the asymmetric unit. The FnIII-3 and FnIII-4 domains exhibit the canonical FnIII fold consisting of two $\beta$-sheets formed by strands $\mathrm{ABE}$ and $\mathrm{C}^{\prime} \mathrm{CFG}$ (strand $\mathrm{G}$ is divided into $\mathrm{G} 1$ and $\mathrm{G} 2$; Figs. $1 b-1 e$ and Supplementary Fig. S2)

A structure of FnIII-3 solved by NMR has been deposited in the PDB (PDB entry 2yrz; RIKEN Structural Genomics/ Proteomics Initiative, unpublished work). Each of the 20 models of the NMR ensemble superposes well on the two FnIII-3 molecules of the crystal structure, with an r.m.s.d. ranging from 0.68 to $0.94 \AA$ for all $\mathrm{C}^{\alpha}$ atoms in the region 1457-1548. In spite of the overall similarity, there are significant differences between the solution and crystal structures (Supplementary Fig. S3). In our crystal structure $\beta$-strand A

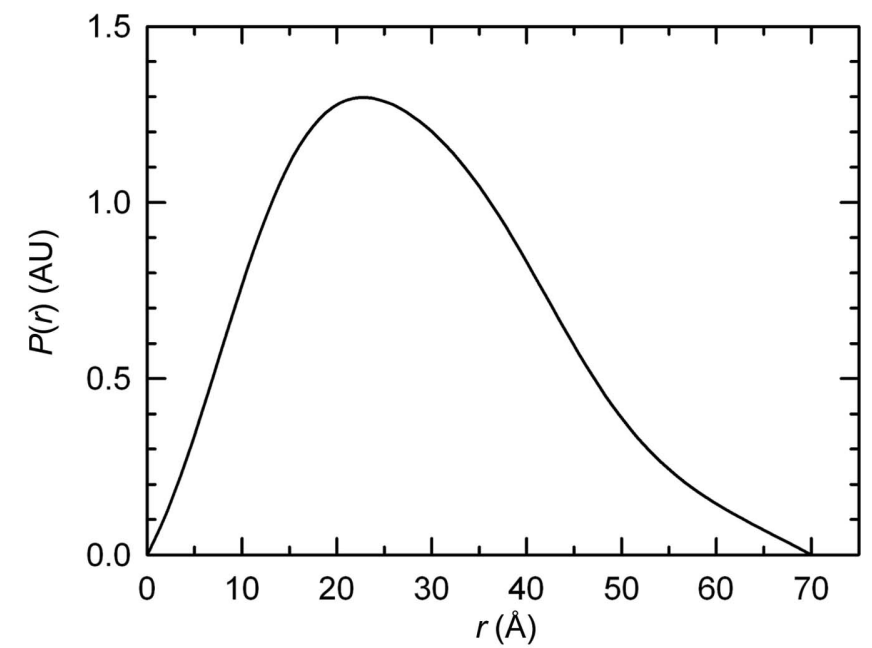

(b)

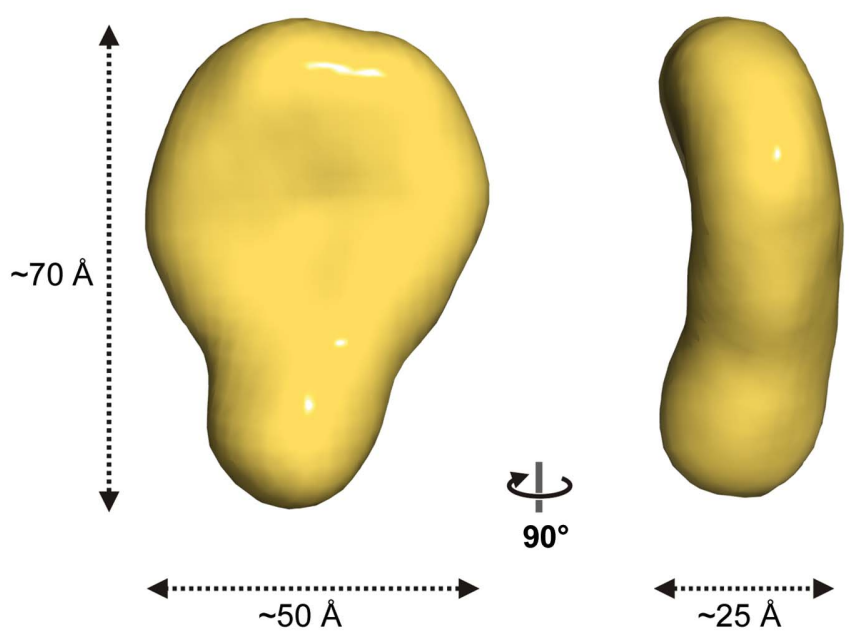

(d)

Figure 3

SAXS analysis of FnIII-3,4 of $\beta 4$. (a) Scattering profile of FnIII-3,4 extrapolated to infinite dilution. The Guinier plot in the range $0.13 \leq q R_{\mathrm{g}} \leq 1.30$ is shown in the inset. (b) $P(r)$ calculated from the SAXS data extending to $0.3 \AA^{-1}$. (c) Dimensionless Krakty plot of the scattering data. The position of the maximum for compact globular particles is indicated by the cross-hair. (d) Orthogonal views of the ab initio molecular envelope obtained with $D A M M I N$; the structure is the average of 19 independent reconstructions. 
extends to A1468, similarly to as observed in other FnIII domains. In contrast, in the NMR structure the carbonyl group of S1467 is flipped and that of A1468 is rotated with respect to the crystal structure, impeding the formation of hydrogen bonds to $\beta$-strand $\mathrm{B}$.

Superimposition of the crystal structures of the four FnIII domains of $\beta 4$ reveals a conserved hydrophobic core that includes nine out of the 11 residues that are identical in the four domains. The polypeptide backbone is highly conserved in the $\beta$-strands and in loops $\mathrm{A} / \mathrm{B}, \mathrm{E} / \mathrm{F}$ and $\mathrm{F} / \mathrm{G} 1$ (Supplementary Fig. S4). On the other hand, the solvent-exposed surfaces of these domains show no similarities (for example, in their electrostatic properties; Supplementary Fig. S4d). In summary, the FnIII domains of $\beta 4$ share a common scaffold, but each of them exhibits distinct external features that support functional specialization.

Y1494 and Y1526 in FnIII-3, which are phosphorylated during signalling, are adjacent in the structure. Their aromatic rings are buried in the core of the domain, while the hydroxyl groups reach a cleft that contains a network of waters that is conserved in the two molecules of the asymmetric unit (Figs. $2 a$ and $2 b$ ). Near Y1526, one of the FnIII-3 molecules has a sulfate ion coordinated by E1501, H1503 and two of the waters in the cleft. This anion is of interest because sulfates frequently mimic the phosphate group of phosphorylated residues. In the other FnIII-3 molecule in the asymmetric unit the position of the sulfate is occupied by the carboxylate of D1519 from a neighbouring molecule in the crystal. Collectively, the pocket near Y1526 has a propensity to bind negatively charged groups, despite being surrounded by the acidic residues E1501, E1518 and D1519. Finally, C1608 and Y1642 in FnIII-4 occupy positions equivalent to Y1494 and Y1526, respectively (Figs. $2 c$ and $2 d$ ). In contrast to FnIII-3, the region around Y1642 is mostly uncharged.

\subsection{Structure of the Fnlll-3,4 region in solution}

Efforts to crystallize the complete FnIII-3,4 region of $\beta 4$ were unsuccessful. Thus, we analyzed this fragment by SAXS, which provides information on the structure in solution at low resolution (Table 2). The scattering profile of FnIII-3,4 (residues 1457-1666) is shown in Fig. 3(a). The radius of gyration $\left(R_{\mathrm{g}}\right)$ determined by Guinier analysis using data at very small scattering angles was $22.1 \pm 0.8 \AA$. The same $R_{\mathrm{g}}$ value was obtained in the calculation of the pairwise distance distribution function, $P(r)$, using data in the range of the scattering vector $(q)$ from 0.01 to $0.30 \AA^{-1}$. The $P(r)$ of FnIII-3,4, which contains information about the size and shape of the particle, has a bell shape characteristic of globular particles and a maximum dimension $\left(D_{\max }\right)$ of $\sim 70 \AA$ (Fig. $3 b$ ).

We used a dimensionless Kratky plot of the SAXS data to analyzed possible interdomain flexibility (Durand et al., 2010). This representation of the FnIII-3,4 data has a maximum of $\sim 1.19$ at $q R_{\mathrm{g}} \simeq 1.90$, which is very close to the expected value for globular compact particles (maximum of $\sim 1.1$ at $q R_{\mathrm{g}} \simeq$ 1.73), suggesting that this protein has very limited flexibility (Fig. 3c).
Molecular envelopes of FnIII-3,4 were constructed from the SAXS data with DAMMIF and DALAI_GA, which implement two different $a b$ initio algorithms. The normalized spatial discrepancy (NSD), a parameter that describes the agreement between three-dimensional models, was $0.67 \pm 0.04$ for 19 reconstructions generated with $D A M M I F$ and $0.98 \pm 0.03$ for 20 models obtained with $D A L A I_{-} G A$. The NSD between the averaged models obtained with $D A M M I F$ and $D A L A I_{-} G A$ was 0.68 . Thus, the two programs yield stable reconstructions of similar shapes. The molecular mass estimated from the excluded volume of the DAMMIF models $(21.0 \mathrm{kDa})$ agrees with the mass calculated from the sequence $(23.4 \mathrm{kDa})$, confirming that FnIII-3,4 is a monomer in solution. The $a b$ initio envelopes resemble a slightly bended and elongated flat disc with overall dimensions of $\sim 70 \times \sim 50 \times \sim 25 \AA$ (Fig. $3 d$ and Supplementary Fig. S5). In summary, analysis of FnIII-3,4
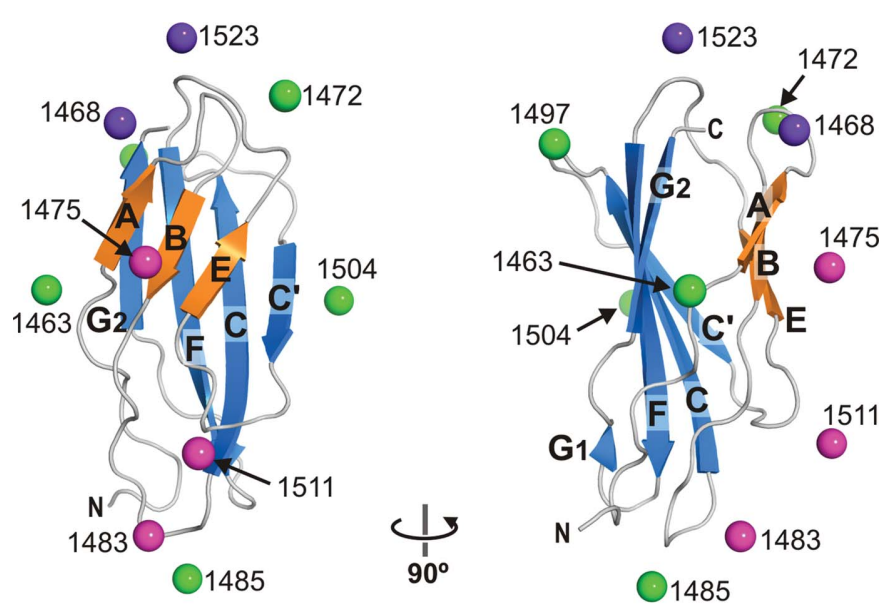

(a)
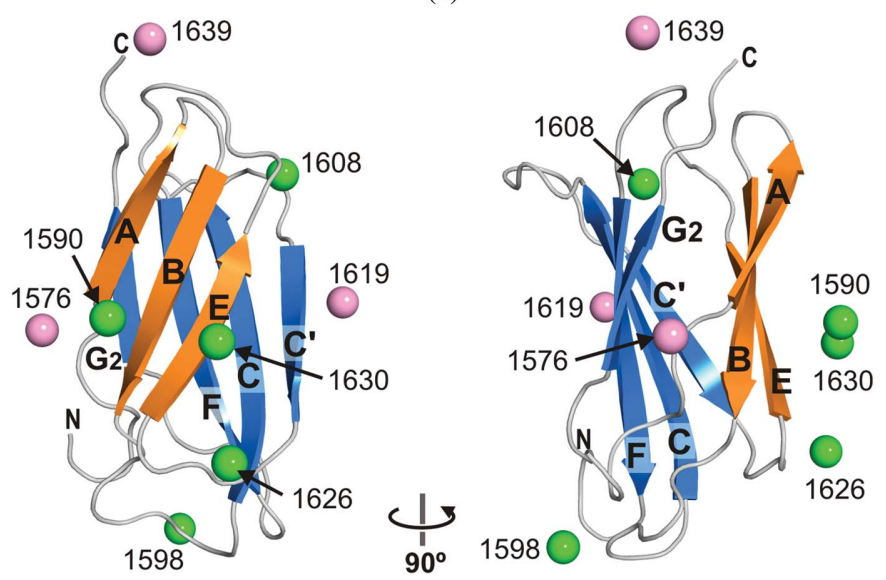

(b)

Figure 4

Paramagnetic labels attached to the FnIII-3 and FnIII-4 domains of $\beta 4$. (a) The predicted average locations of the nitroxide group of MTSL attached to Cys are shown as spheres in the crystal structure of FnIII-3. The positions that yielded well defined inter-spin distances with a second probe at $\mathrm{C} 1608$ are coloured green. Those that resulted in broad distance distributions but did not alter the global structure of FnIII-3,4 are coloured magenta. The positions that resulted in a distortion of the structure are coloured dark violet. (b) Crystal structure of FnIII-4 with the estimated average position of the paramagnetic groups. The positions that yielded useful distances are coloured green, while those that resulted in broad distributions are shown in pink. 
Table 2

SAXS data collection and derived parameters for $\beta 4$ FnIII-3,4.

\begin{tabular}{|c|c|}
\hline \multicolumn{2}{|l|}{ Data-collection parameters } \\
\hline Instrument & $\begin{array}{l}\text { EMBL P12 beamline, PETRA, } \\
\text { DESY, Hamburg }\end{array}$ \\
\hline Beam geometry & $0.2 \times 0.12 \mathrm{~mm}$ \\
\hline Wavelength $(\AA)$ & 1.24 \\
\hline$q$ range $\left(\AA^{-1}\right)$ & $0.01-0.35$ \\
\hline Exposure time (s) & $20 \times 0.05$ \\
\hline Concentration range $\left(\mathrm{mg} \mathrm{ml}^{-1}\right)$ & $1.5-48.6$ \\
\hline Temperature (K) & 283 \\
\hline \multicolumn{2}{|l|}{ Structural parameters $\dagger$} \\
\hline$I(0) / c \ddagger\left(10^{-2} \mathrm{~cm}^{2} \mathrm{mg}^{-1}\right)$ (from Guinier) & $1.24 \pm 0.01$ \\
\hline$R_{\mathrm{g}}(\AA)$ (from Guinier) & $22.1 \pm 0.8$ \\
\hline$I(0) \ddagger\left(10^{-2} \mathrm{~cm}^{2} \mathrm{mg}^{-1}\right)[$ from $P(r)]$ & $1.23 \pm 0.01$ \\
\hline$R_{\mathrm{g}}(\AA)[$ from $P(r)]$ & 22.0 \\
\hline$D_{\max }(\AA)$ & 70 \\
\hline Porod volume estimate $V_{\mathrm{n}}\left(\AA^{3}\right)$ & $32000 \pm 1000$ \\
\hline Excluded volume estimate $V_{\mathrm{ex}}\left(\AA^{3}\right)$ & $42000 \pm 200$ \\
\hline \multicolumn{2}{|l|}{ Molecular-mass determination } \\
\hline$I(0) \ddagger\left(10^{-2} \mathrm{~cm}^{2} \mathrm{mg}^{-1}\right)$ & $4.20 \pm 0.01$ \\
\hline Molecular mass $M_{\mathrm{r}} \S(\mathrm{Da})[$ from $I(0)]$ & 19200 \\
\hline Molecular mass $M_{\mathrm{r}} \S(\mathrm{Da})\left[\right.$ from $\left.\left(V_{\mathrm{p}} 1.6\right)\right]$ & $20000 \pm 625$ \\
\hline Molecular mass $M_{\mathrm{r}} \S(\mathrm{Da})\left[\right.$ from $\left.\left(V_{\mathrm{ex}} / 2\right)\right]$ & $21000 \pm 100$ \\
\hline Calculated monomeric $M_{\mathrm{r}}$ from sequence & 23360 \\
\hline \multicolumn{2}{|l|}{ Software used } \\
\hline Primary data reduction & Automated SAXS pipeline \\
\hline Data processing & PRIMUS (Konarev et al., 2003) \\
\hline Ab initio analysis & $\begin{array}{l}\text { DAMMIF (Franke \& Svergun, } \\
\text { 2009), DALAI_GA } \\
\text { (Chacón et al., 2000) }\end{array}$ \\
\hline Validation and averaging & $\begin{array}{l}\text { SUPCOMB (Kozin \& Svergun, } \\
\text { 2001) DAMAVER (Volkov } \\
\text { \& Svergun, 2003) }\end{array}$ \\
\hline Computation of model intensities & CRYSOL (Svergun et al., 1995) \\
\hline $\begin{array}{l}\text { Three-dimensional graphical } \\
\text { representations }\end{array}$ & PyMOL (Schrödinger) \\
\hline SASBDB code & SASDAT6 \\
\hline
\end{tabular}

$\dagger$ Values reported for the data extrapolated to infinite dilution. $\$$ Absolute intensities determined using water as a secondary standard (Orthaber et al., 2000). § Calculated using BSA as a standard (66 $000 \mathrm{Da})$.

by SAXS revealed a compact structure in which the two FnIII domains might establish lateral interactions and have their longitudinal axes approximately lying on the same plane.

3.3. Determination of interdomain distances in the FnIII-3,4 region by SDSL and DEER using doubly spin-labelled samples

Owing to the globular shape of the individual domains, it was not possible to unambiguously dock the crystal structures of FnIII-3 and FnIII-4 into the SAXS-derived envelopes or to locate them by rigid-body fitting against the SAXS data. Therefore, to gather information on the relative orientation of the two FnIII domains we used EPR spectroscopy, since DEER experiments in combination with SDSL allow the determination of distances between selected residues in the range $\sim 10-80 \AA$ (Jeschke, 2012), which fits the dimensions of the protein. We attached the MTSL paramagnetic probe to Cys either present in the integrin or engineered at solventexposed positions of FnIII-3 and FnIII-4 (Fig. 4 and Supplementary Figs. S6 and S7).

$\beta 4$ contains three Cys residues in the FnIII-3,4 region: C1483 in FnIII-3, C1559 in the linker that connects the two FnIII domains and C1608 in FnIII-4. MTSL reacted with these three Cys residues when the wild-type protein was labelled.
To measure multiple interdomain distances between MTSLs attached to pairs of selected positions, each in one FnIII domain, we initially attempted to substitute the three wild-type Cys residues. The triple mutants C1483A/C1559A/ C1608A and C1483S/C1559A/C1608S were insoluble when expressed in E. coli. On the other hand, the double mutant C1483S/C1559A was produced as a soluble protein and was
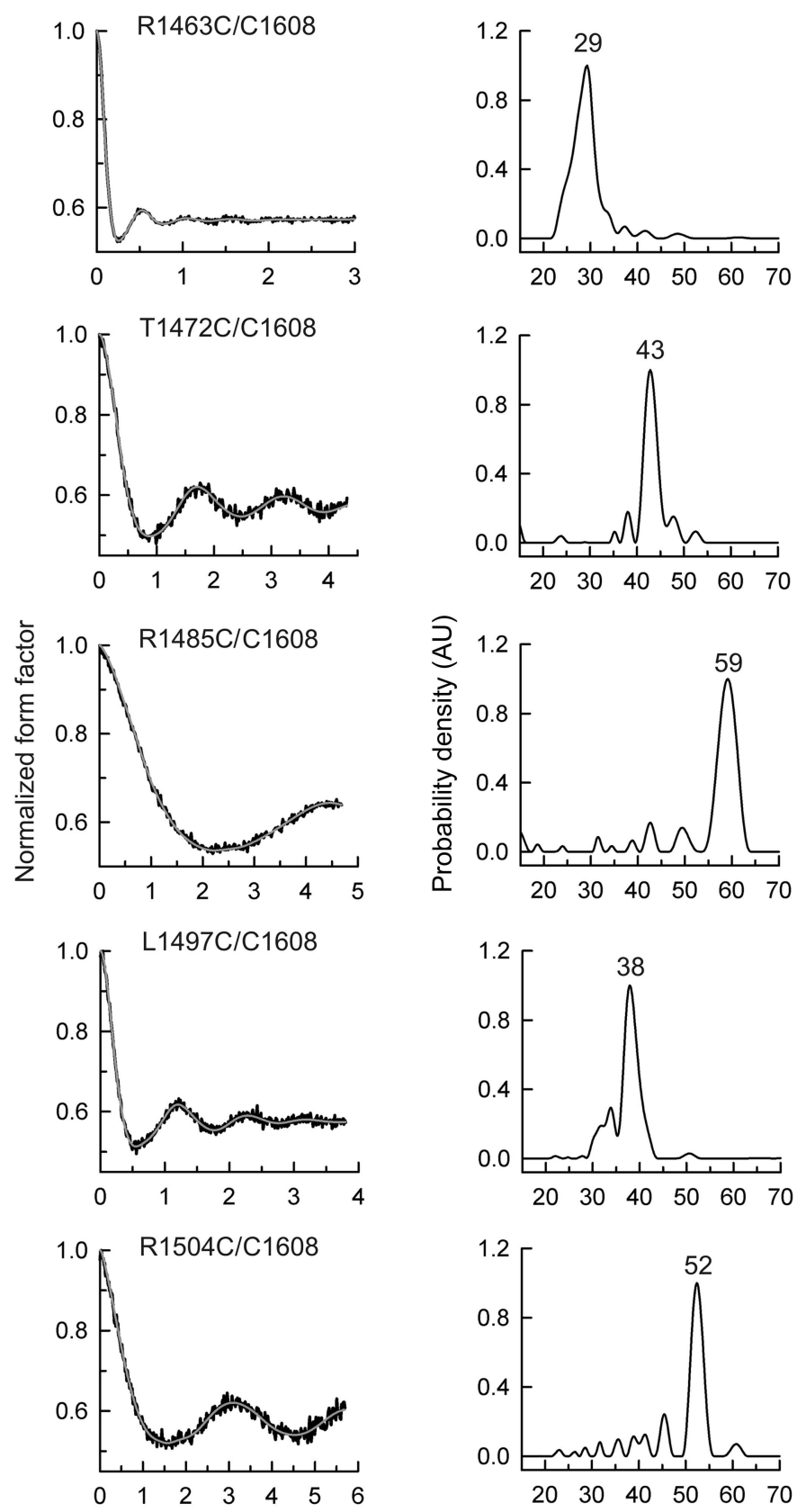

Dipolar evolution time ( $\mu \mathrm{s})$

(a)

Inter-spin distance $(\AA)$

(b)

Figure 5

DEER results for the doubly labelled FnIII-3,4 mutants used to gather distances between C1608 in FnIII-4 and five positions in FnIII-3. (a) Normalized dipolar evolution (black lines) and fits to the data (grey lines) for each double Cys mutant. (b) Inter-spin distance distribution profiles calculated from the data in $(a)$. The distances of the major peaks are indicated at the top. 
Table 3

Inter-spin distances and structural parameters of FnIII-3,4 mutants labelled with MTSL.

\begin{tabular}{|c|c|c|c|c|c|c|}
\hline \multirow[b]{2}{*}{ FnIII-3,4 mutant } & \multicolumn{3}{|c|}{ Spin-labelled sites } & \multirow[b]{2}{*}{$\begin{array}{l}\text { Average inter-spin } \\
\text { distance }(\AA)\end{array}$} & \multicolumn{2}{|c|}{$\begin{array}{l}\text { Structural } \\
\text { parameters } \dagger\end{array}$} \\
\hline & FnIII-3 & Linker & FnIII-4 & & $\begin{array}{l}R_{\mathrm{g}} \\
(\mathrm{A})\end{array}$ & $\begin{array}{l}D_{\max } \\
(\AA)\end{array}$ \\
\hline $\mathrm{R} 1463 \mathrm{C} / \mathrm{C} 1608 \neq$ & 1463 & - & 1608 & 29 & 22.5 & 70 \\
\hline $\mathrm{T} 1472 \mathrm{C} / \mathrm{C} 1608 \ddagger$ & 1472 & - & 1608 & 43 & 22.6 & 70 \\
\hline $\mathrm{R} 1485 \mathrm{C} / \mathrm{C} 1608$ 丰 & 1485 & - & 1608 & 59 & 22.2 & 70 \\
\hline L1497C/C1608\$ & 1497 & - & 1608 & 38 & 22.7 & 70 \\
\hline R1504C/C1608末 & 1504 & - & 1608 & 52 & 22.3 & 70 \\
\hline \multirow[t]{3}{*}{ L1497C/N1598C/C1608 } & - & - & 1598,1608 & $30(27 / 34) \S$ & 22.6 & 70 \\
\hline & 1497 & - & 1598 & 14 & & \\
\hline & 1497 & - & 1608 & 37 & & \\
\hline \multirow{3}{*}{$\mathrm{R} 1504 \mathrm{C} / \mathrm{N} 1598 \mathrm{C} / \mathrm{C} 1608 \ddagger$} & - & - & 1598,1608 & $28 / 35(27 / 34) \S$ & 22.3 & 70 \\
\hline & 1504 & - & 1608 & 52 & & \\
\hline & 1504 & - & 1598 & 25 & & \\
\hline \multirow[t]{3}{*}{ T1472C/S1590C/C1608 } & - & - & 1590,1608 & $27(25) \S$ & 22.7 & 70 \\
\hline & 1472 & - & 1608 & 43 & & \\
\hline & 1472 & - & 1590 & 27 & & \\
\hline \multirow[t]{3}{*}{ T1472C/C1608/S1626C $\ddagger$} & - & - & 1626,1608 & $28(28) \S$ & 22.8 & 70 \\
\hline & 1472 & - & 1608 & 42 & & \\
\hline & 1472 & - & 1626 & $33 / 39$ ब & & \\
\hline \multirow{3}{*}{ L1497C/C1608/S1626C $\ddagger$} & - & - & 1626,1608 & $28 / 30(28) \S$ & 22.8 & 70 \\
\hline & 1497 & - & 1608 & 38 & & \\
\hline & 1497 & - & 1626 & $24 / 28 / 30$ & & \\
\hline \multirow[t]{3}{*}{$\mathrm{R} 1504 \mathrm{C} / \mathrm{C} 1608 / \mathrm{S} 1626 \mathrm{C} \ddagger$} & - & - & 1626,1608 & $29 / 32(28) \S$ & 22.7 & 70 \\
\hline & 1504 & - & 1608 & 52 & & \\
\hline & 1504 & - & 1626 & $42 / 46-$ & & \\
\hline \multirow[t]{3}{*}{ T1472C/C1608/R1630C $\ddagger$} & - & - & 1630,1608 & $23(23) \S$ & 22.7 & 70 \\
\hline & 1472 & - & 1608 & 43 & & \\
\hline & 1472 & - & 1630 & $33 / 36 / 40$ - & & \\
\hline \multirow[t]{3}{*}{$\mathrm{R} 1504 \mathrm{C} / \mathrm{C} 1608 / \mathrm{R} 1630 \mathrm{C} \ddagger$} & - & - & 1630,1608 & $23(23) \S$ & 22.6 & 70 \\
\hline & 1504 & - & 1608 & 54 & & \\
\hline & 1504 & - & 1630 & $42 / 49$ & & \\
\hline $\mathrm{R} 1485 \mathrm{C} / \mathrm{C} 1559+\dagger$ & 1485 & 1559 & - & 51 & 22.3 & 70 \\
\hline $\mathrm{C} 1559 / \mathrm{C} 1608$ 米 & - & 1559 & 1608 & 45 & 21.7 & 70 \\
\hline
\end{tabular}

$\dagger$ Determined from the SAXS profile by indirect transform using data for $q \leq 0.3 \AA^{-1}$. $\neq$ Contains the substitutions C1483S and C1559A. § Numbers in parentheses indicate the position of the maximum of the distance distribution estimated by modelling the MTSL probe on the crystal structure of the FnIII-4 domain. For distances with multimodal distributions, which are likely to correspond to alternative conformations of the probe, the mean value was used for rigid-body fitting. I† Contains the substitutions C1483A and C1559A. \$t Contains the single mutation C1483A.

used as a template to create nine triple $\beta 4$ mutants that contain the wild-type C1608 and a second Cys engineered at a solvent-exposed position of FnIII-3. The purpose of this series of mutants was to locate C1608 with respect to FnIII-3. For clarity, we refer to the FnIII-3,4 mutants by the position of the Cys residue present. EPR analysis of the R1463C/C1608, T1472C/C1608, R1485C/C1608, L1497C/C1608 and R1504C/ C1608 mutants yielded distance distributions that were characterized by single major narrow peaks centred at 29, 43, 59, 38 and $52 \AA$, respectively (Fig. 5, Table 3). This indicates that the spin label at residue 1608 occupies a relatively fixed position with respect to FnIII-3, suggesting low conformational variability of the label (as predicted by modelling; see Supplementary Fig. S7) and very limited interdomain wiggling. SAXS analysis of these mutants labelled with MTSL revealed that they have the same overall structural parameters as the wild-type protein (Supplementary Figs. S8a and S8b), confirming that the mutations and subsequent labelling did not distort the global structure of the fragment.

Two other mutants in this series, R1475C/C1608 and A1511C/C1608, showed SAXS-derived $R_{\mathrm{g}}, D_{\max }$ and $P(r)$ values similar to those of wild-type FnIII-3,4, yet DEER analysis revealed broad inter-spin distance distributions (Supplementary Figs. S $8 c-\mathrm{S} 8 f$ ) that were not used for structural modelling of the fragment. Finally, SAXS analysis of two additional mutants, A1468C/ C1608 and N1523C/C1608, showed significantly larger $R_{\mathrm{g}}$ (26.1 and $26.1 \AA$ ) and $D_{\text {max }}(\sim 88$ and $\sim 92 \AA)$ values than the wild type and an apparently large flexibility (Supplementary Figs. S8c$\mathrm{S} 8 g$ ), which suggests a distorted interdomain arrangement. Furthermore, DEER analysis of these mutants showed multiple inter-spin distance peaks and consequently these distances were not used for rigid-body fitting. In summary, Ala1468 and Asn1523, which are $\sim 14 \AA$ apart on the FnIII-3 surface, define an area that is important for maintaining the organization of the FnIII-3,4 structure.

Next, we attempted to obtain the position of C1483 in FnIII-3 with respect to FnIII-4 following the same approach. For this, we created mutants with the C1608A substitution, which had C1483 and an engineered Cys at solvent-exposed positions of FnIII-4 (Supplementary Table S1). However, DEER analysis of the mutants yielded broad and multimodal distance distributions (Supplementary Fig. S9). Modelling of MTSL attached to C1483 with $M M M$ indeed confirms that labelling of this residue is unfavourable and could distort the structure. Nevertheless, replacing the label at the C1483 position by a label at $\mathrm{R} 1485 \mathrm{C}$ did not solve the problem because the protein mutants were either insoluble or yielded very wide inter-spin distance distributions (Supplementary Fig. S9).

\subsection{Determination of additional interdomain distances by DEER using triple spin-labelled FnIII-3,4 mutants}

In case $\mathrm{C} 1608$ was important to preserve the native-like structure, we decided to maintain this residue and designed mutants that contain three Cys residues and carry C1483S/ C1559A substitutions. These proteins include a Cys pair of C1608 in FnIII-4 and a Cys in FnIII-3 which had yielded well defined inter-spin distances in the double-labelled mutants. In addition, they contain a new Cys engineered at solventexposed positions of FnIII-4. We generated the eight triple Cys mutants collected in Table 3. All of these proteins were produced in a soluble form; they were labelled with MTSL and analyzed by SAXS and DEER. Analysis of the SAXS profiles 
of these mutants labelled with MTSL revealed that they are monomeric in solution and that they have an overall structure compatible with that of the wild-type protein (Supplementary Figs. S10 $a$ and S10b).

Analysis of the triply labelled mutants by DEER is expected to yield information on three inter-spin distances. Firstly, an intradomain distance between the probes at C1608 and at a second Cys in FnIII-4; secondly, an interdomain distance between the probes attached to each of the engineered Cys residues in FnIII-3 and C1608 in FnIII-4; and finally, a second interdomain distance between the probes attached at the Cys engineered in both domains, which is the distance that we want to use to determine the orientation of the domains. In addition to the three inter-spin distances, DEER measurements of objects containing three spin labels have been reported to show artifacts or ghost distances (von Hagens et al., 2013). In order to avoid and identify the presence of such artifacts, the measurements were performed at lower inversion efficiencies for the pump pulse of $\mathrm{mw}_{2}$
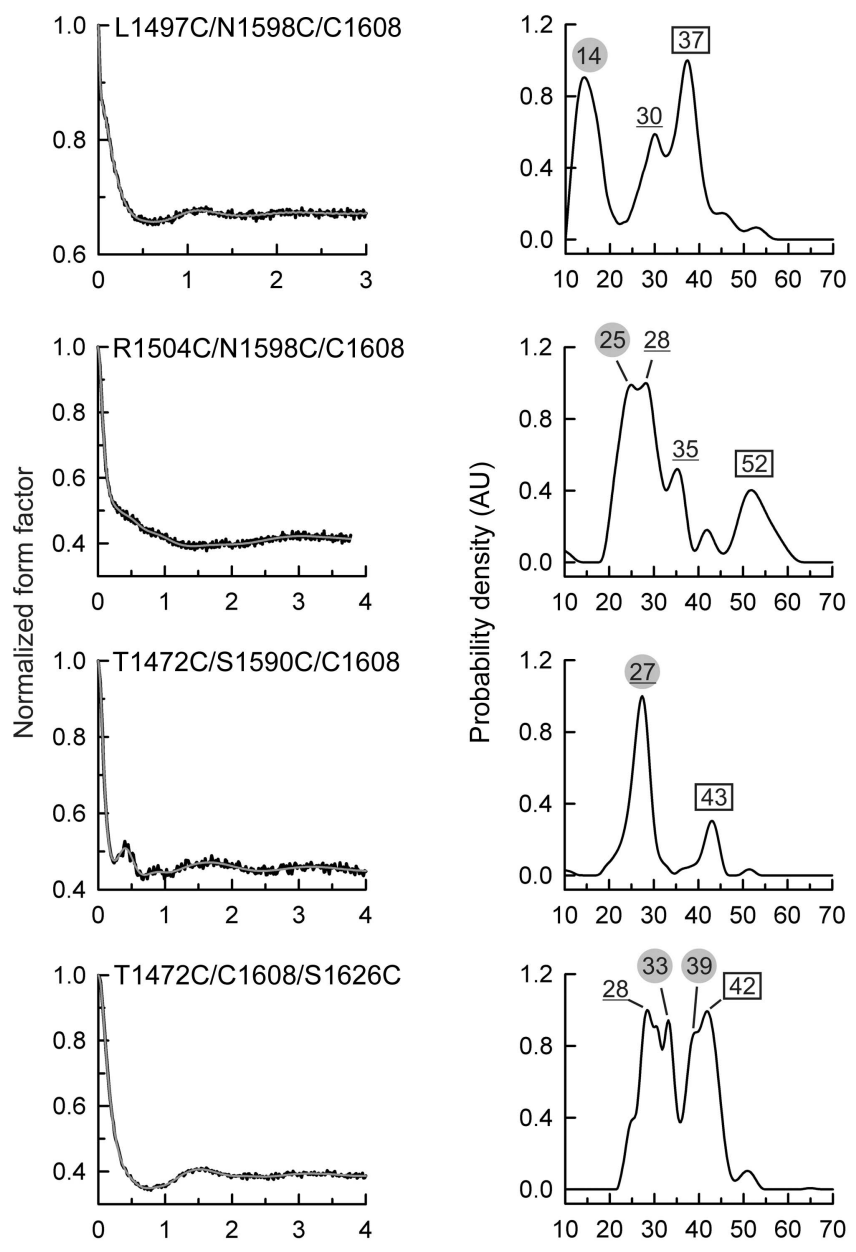

Dipolar evolution time $(\mu \mathrm{s})$

(a)

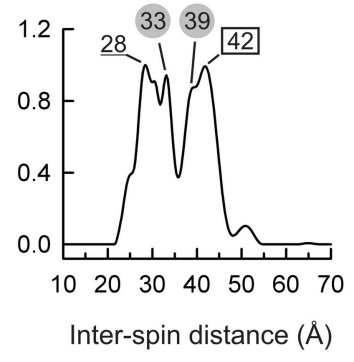

(b) (pump pulse of turning angle $\pi, \pi / 2$ and $\pi / 4$ ). No ghost peaks could be identified for any of the samples; for this reason, the spectra shown in Fig. 6 are those obtained with the normal DEER sequence containing a $\pi$ pump pulse.

In general, the DEER spectra of the triply labelled mutants showed several peaks that were broader than in the doubly labelled mutants. In order to identify the interdomain distance of interest, the peaks corresponding to the other distances were assigned. The FnIII-4 intradomain distances (underlined values in Fig. 6) were estimated by modelling the conformation of the probes with $M M M$ using the crystal structure of FnIII-4. The inter-domain distances involving C1608 (values in square boxes in Fig. 6) could be identified from the measurements of double-labelled mutants. By exclusion, the remaining peaks could be assigned to new interdomain distance distributions (values surrounded by circles in Fig. 6). Whenever two or more peaks were assigned to the interdomain distance not involving $\mathrm{C} 1608$, an average value for the distance was taken for the structure modelling.
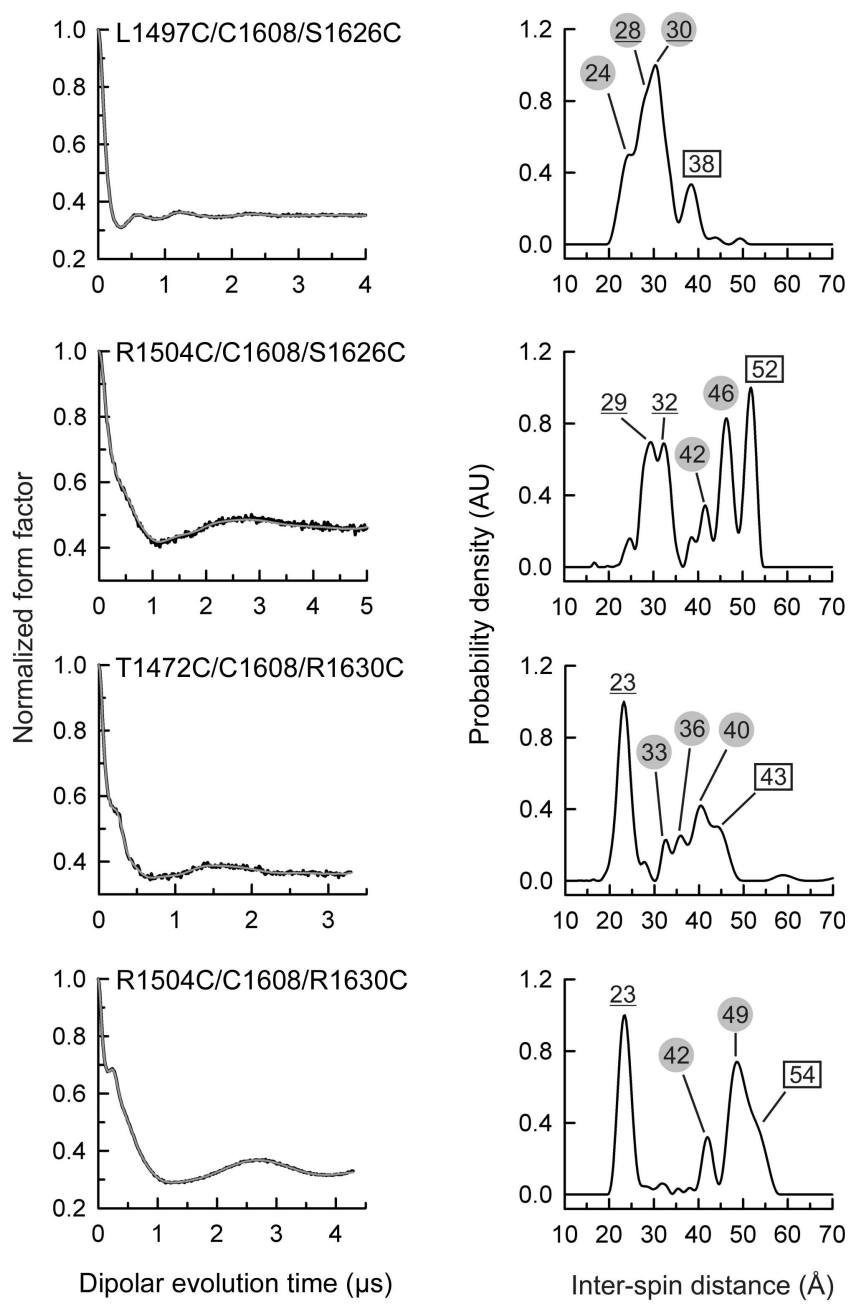

(a)

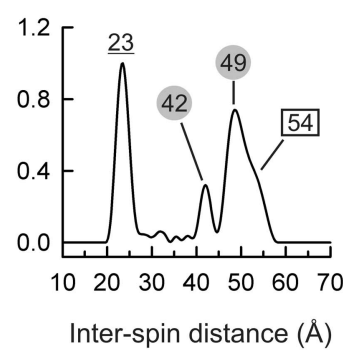

(b)

Figure 6

DEER results for triply labelled FnIII-3,4 mutants. (a) Normalized dipolar evolution (black lines) and fits to the data (grey lines) for each of eight triply labelled mutants. $(b)$ Distance distribution profiles calculated from the data in $(a)$. The positions of peaks that correspond to distances previously observed in doubly labelled mutants containing C1608 are shown in rectangles. The positions of peaks assigned to intradomain distances modelled on the FnIII-4 structure are underlined. Finally, the peaks attributed to interdomain distances that do not involve C1608 are shown in grey circles. See Supplementary Fig. S10 for analysis of these proteins by SAXS. 
Peaks in the DEER inter-spin distance distributions of the eight triply labelled mutants were assigned to pairs of labelling positions as follows. The inter-spin distance distribution of the L1497C/N1598C/C1608 mutant shows three peaks at $\sim 14, \sim 30$ and $\sim 37 \AA$. Modelling of MTSL at 1598-1608 within FnIII-4 predicts a bimodal inter-spin distance at 27 and $34 \AA$, which is likely to correspond to the observed peak centred at $30 \AA$. The experimentally observed distance between the 1497-1608 pair was $37 \AA$. Hence, the distance peak at $\sim 14 \AA$ was assigned to the 1497-1598 pair.

The distance distribution obtained for the R1504C/N1598C/ C1608 mutant shows peaks at 25, 28, 35 and $52 \AA$. As described above, the peaks at 28 and $35 \AA$ were assigned to the intradomain pair $1598-1608$. The peak at $52 \AA$ corresponds to the 1504-1608 pair. Thus, the peak at $25 \AA$ was assigned to the 1504-1598 pair.

The T1472C/S1590C/C1608 mutant yielded a distance distribution with two peaks at 27 and $43 \AA$. The first peak corresponds to the estimated distance between the probes at residues 1590 and 1608 , while the spacing at $43 \AA$ had previously been observed for the 1472-1608 pair. Owing to the large integrated intensity of the peak at $27 \AA$, this distance was also assigned to the $1472-1590$ pair.

The distance distribution of the T1472C/C1608/S1626C mutant has peaks at $28,33,39$ and $42 \AA$. The distance between the intradomain pair 1608-1626 was estimated to be $28 \AA$. The $42 \AA$ peak corresponds to the $1472-1608$ pair. Therefore, the doublet at 33 and $39 \AA$ was assigned to the $1472-1626$ pair.

The distance distribution of the L1497C/C1608/S1626C mutant has peaks at 24,30 and $38 \AA$ and a shoulder at $\sim 28 \AA$. The estimated distance between the $1608-1626$ pair $(28 \AA)$ is likely to correspond to the peaks at $28-30 \AA$. The distance

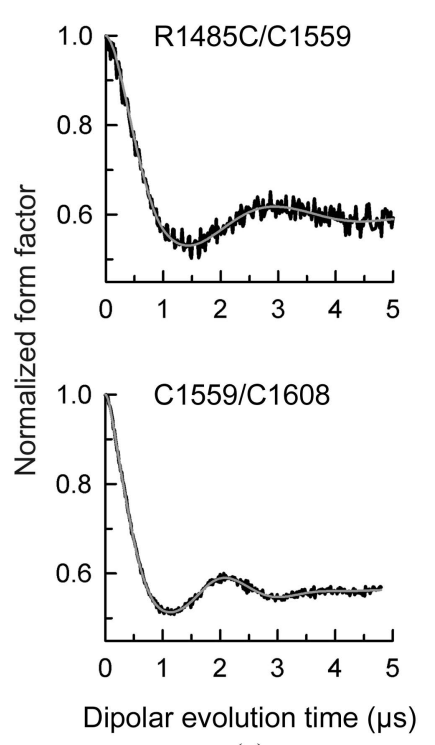

(a)

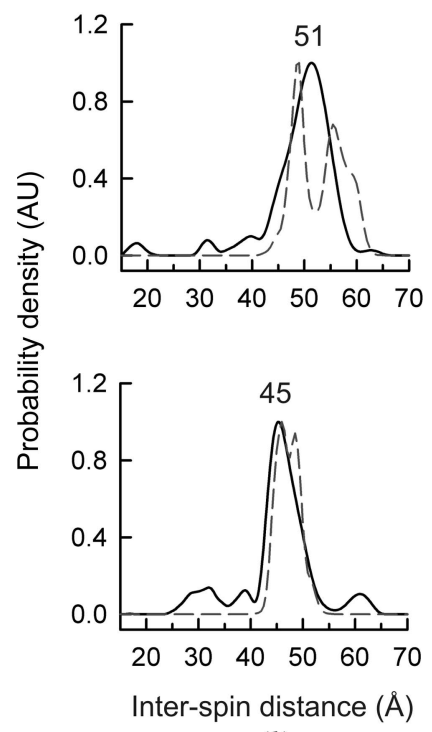

(b)
Figure 7

DEER results for mutants containing $\mathrm{C} 1559$ in the interdomain linker. (a) Normalized dipolar evolution (black lines) and fits to the data (grey lines) for two doubly labelled mutants that include C1559. (b) Distance distribution profiles calculated from the data in (a) (solid line) and a calculated distribution for one of the final models (dashed line). See Supplementary Fig. S10 for analysis of these proteins by SAXS. between the 1497-1608 pair was already observed at $38 \AA$ in the doubly labelled mutant (L1497C/C1608). Finally, the pair $1497-1626$ was assigned to the triplet at 24,28 and $30 \AA$.

The distance distribution of the R1504C/C1608/S1626C mutant has peaks at 29 and $32 \AA$ that are similar to the estimated distance between the probes modelled at residues 1608 and 1626 in FnIII-4. There is also a peak at $52 \AA$ that corresponds to the distance measured for the 1504-1608 pair. Finally, two peaks at 42 and $46 \AA$ were attributed to the 1504 1626 pair.

The T1472C/C1608/R1630C mutant produced a distance distribution with a major peak at $23 \AA$ and several peaks in the range 33-43 $\AA$. The distance at $23 \AA$ matches the estimated separation of the nitroxide groups of the pair 1608-1630. On the other hand, the 1472-1608 pair yields a distance peak at $43 \AA$ in previous doubly and triply labelled mutants. Hence, the triplet at 33,36 and $40 \AA$ was assigned to the distance of the 1472-1630 pair.

Finally, the distance distribution obtained for the R1504C/ C1608/R1630C mutant shows the peak at $23 \AA$ attributed to the 1608-1630 pair and peaks at 42 and $49 \AA$. The distance of the 1504-1608 pair, expected to be $52 \AA$, appears as a shoulder at $54 \AA$. Therefore, the doublet at 42 and $49 \AA$ was assigned to the 1504-1630 pair.

In summary, analysis of the triply MTSL-labelled FnIII-3,4 proteins yielded eight nonredundant interdomain distances between the spin probe attached to pairs of residues different from C1608. All distances and their assignments are collected in Table 3.

3.5. DEER measurement of distances between C1559 in the interdomain linker and the FnIII domains

To obtain information on the position of the linker that connects the two FnIII domains, we measured distances between MTSL probes attached to C1559, located at the centre of the linker, and to a second Cys in either FnIII-3 or FnIII-4. We used the single mutant C1483A that contains the Cys pair C1559/C1608 present in the wild-type sequence and the triple mutant C1483A/R1485C/C1608A that contains the pair R1485C/C1559. The structural parameters estimated from the SAXS profiles of these two mutants labelled with MTSL revealed that they have similar $R_{\mathrm{g}}, D_{\max }$ and $P(r)$ values to the wild-type protein (Supplementary Figs. S10c and S10d). The distance distributions derived from the DEER data of these two mutants are characterized by single major peaks centred at $51 \AA$ (R1485C/C1559) and $45 \AA$ (C1559/C1608) (Fig. 7 and Table 3 ) that can be explained by considering a fixed anchor for the probe at $\mathrm{C} 1559$, indicating that the linker might have very moderate flexibility.

3.6. Modelling the structure of the FnIII-3,4 region by combining crystal structures, SAXS and DEER-derived distance constraints

13 interdomain distances derived from the DEER measurements (Table 3) were used to derive the relative position of the FnIII-3 and FnIII-4 domains (Supplementary 
Fig. S11). Each distance value in the table corresponds to the average distance between the paramagnetic groups in all possible conformations of the MTSLs at the two labelled positions. Owing to the large conformational flexibility of the probe, for a single labelling site the paramagnetic groups could occupy positions more than $1 \mathrm{~nm}$ apart from each other. To reduce the uncertainty in the location of the $\mathrm{N}-\mathrm{O}$ group, the conformation distribution of MTSL was computed for every labelled position using a rotamer-library approach and the crystal structures of the individual domains (Supplementary Fig. S6 and S7); the average coordinates of the paramagnetic centre were then calculated.

To explore the space of relative arrangement between the two FnIII domains, FnIII-3 was fixed and the position of FnIII-4 was swept using an exhaustive six-dimensional search. Only orientations with a discrepancy between the calculated and experimental DEER distances (parameter $\sigma_{\text {DEER }}$ ) of $\leq 3 \AA$ were accepted. This produced a set of relative arrangements compatible with the DEER restraints. These structures were grouped into 24 clusters defined by a maximum r.m.s.d. of $4 \AA$ for the position of all of the backbone atoms within each cluster, from which one representative structure was selected. Then, for each representative structure, multiple conformations of the 23-residue linker that connects the FnIII-3 and FnIII-4 domains were built using native-like geometry. For every resulting model of the FnIII-3,4 region, the SAXS profile and the parameter $\sigma_{\text {DEER }}$ were calculated, including the two DEER-distance restraints involving C1559. The plausible models were selected if the parameter $\chi_{\mathrm{SAXS}}$, the discrepancy between the calculated and the experimental SAXS curves, was below 1.5 and $\sigma_{\text {DEER }}$ was $\leq 3 \AA$ (Figs. $8 a$ and $8 b$ ). It turned out that of 21 selected models for the structure of the complete fragment, 18 of them correspond to the same relative arrangement of FnIII-3 and FnIII-4, differing only in the structure of the linker. The resulting composite atomic structures fit into the reconstructions created ab initio from the SAXS data, the scattering curves calculated for the models match the SAXS profile and the calculated $P(r)$ of the models reproduce the distribution estimated from the experimental scattering curve (Figs. $8 c, 8 d$ and $8 e$ ).
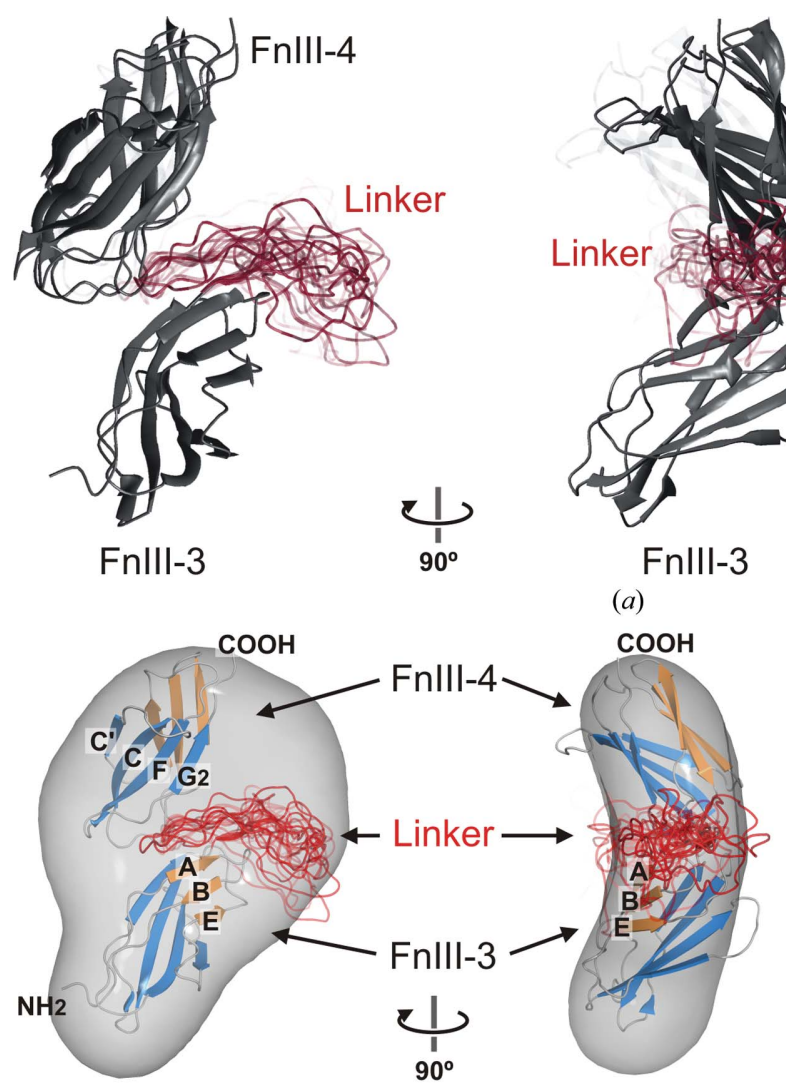

(c)

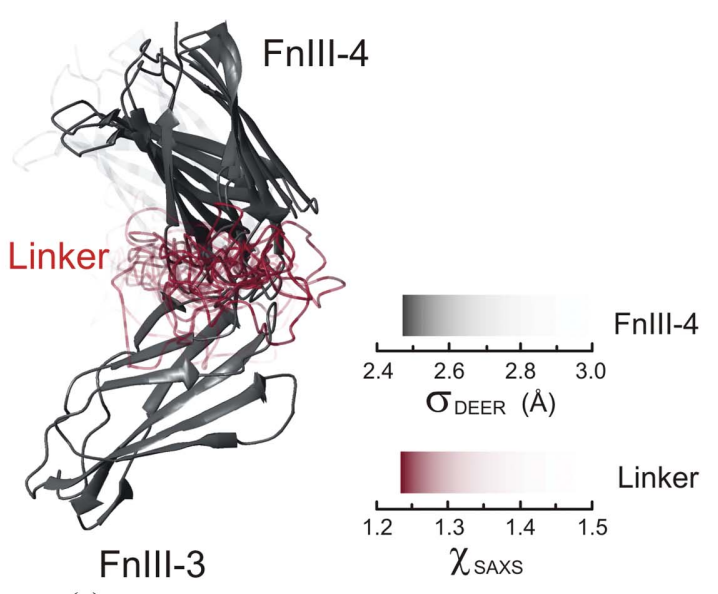

(a)

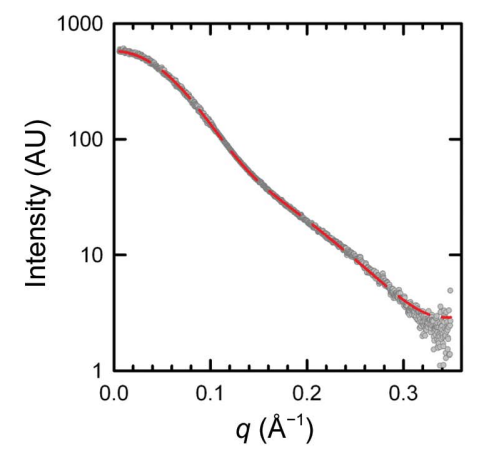

(d)

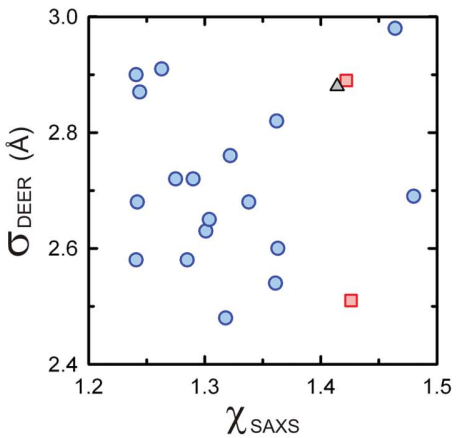

(b)

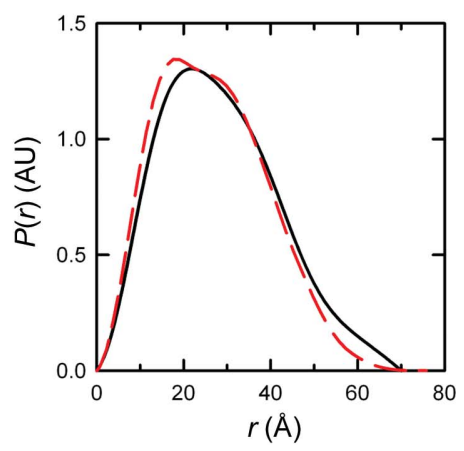

(e)

Figure 8

Structure of the FnIII-3,4 region derived from a combination of crystal structures, DEER, SAXS and modelling methods. (a) Two views of the ensemble of models of FnIII-3,4 that fit the DEER and SAXS data. The orientations of FnIII-4 are coloured according to the fit to the 15 DEER restraints $\left(\sigma_{\text {DEER }}\right)$ of the best models once the linker had been built. The colour intensity of the models of the linker (red) denotes the fit to the SAXS data ( $\chi_{\mathrm{SAXS}}$ ) of the FnIII-3,4 structures. The $\sigma_{\text {DEER }}$ and $\chi_{\text {SAXS }}$ colour scales are shown. $(b)$ Plot of the $\sigma_{\text {DEER }}$ and $\chi_{\text {SAXs }}$ values determined for the 21 models that best fit to the experimental data. $\chi_{\mathrm{SAXS}}$ was determined for $q \leq 0.3 \AA^{-1}$. Points with the same symbol correspond to models with the same orientation of FnIII- 4 . The SAXS profile is sensitive to the conformation of the linker, as revealed by the wide spread of $\chi_{\text {saxs }}$ values for a given arrangement of the two FnIII domains. (c) Docking of the atomic models to the SAXS-derived averaged molecular envelope. For clarity, only the most frequent FnIII-4 orientation and the corresponding modelled linkers are shown. $(d)$ Comparison of the theoretical scattering curve of the model with lowest $\chi_{\text {saxs }}$ (red dashed line) to the experimental SAXS data (grey dots). (e) $P(r)$ functions calculated for the atomic model (red dashed line) and estimated from the SAXS data (black line). Details of the modelling of the inter-domain orientation are shown in Supplementary Fig. S11. 


\subsection{Structure of FnIII-3,4 of integrin $\beta 4$}

The relative orientations of the FnIII-3 and FnIII-4 domains were similar in the refined models. The longitudinal axes of the two domains form an angle of $\sim 170^{\circ}$, resulting in a slightly bent structure. The ABE $\beta$-sheet of FnIII-3 and the $\mathrm{C}^{\prime} \mathrm{CFG} \beta$-sheet of FnIII-4 are oriented towards the concave or inner side of the structure. Strands A and G2 at one edge of the $\beta$-sandwich of FnIII-3 face a region of FnIII-4 formed by the $\mathrm{B} / \mathrm{C}$ and $\mathrm{F} / \mathrm{G} 2$ loops and the N-terminus.

The linker occupies a region near the $\mathrm{A} / \mathrm{B}$ and $\mathrm{E} / \mathrm{F}$ loops at one tip of FnIII-3. Notably, A1468 and N1523, substitution of which by Cys-MTSL distorted the FnIII-3,4 structure (see above), are located in strand $\mathrm{A}$ and loop E/F of FnIII-3, respectively, near the estimated position of the linker. Thus, mutation of A1468 and N1523 is likely to distort the structure by altering primarily the organization of the linker. The modelled position of the linker and the small contact between the two domains support the linker being required for the correct arrangement of the FnIII-3 and FnIII-4 domains.

FnIII-3,4 mediates interactions with several proteins, yet no specific binding sites have been mapped in this region. The

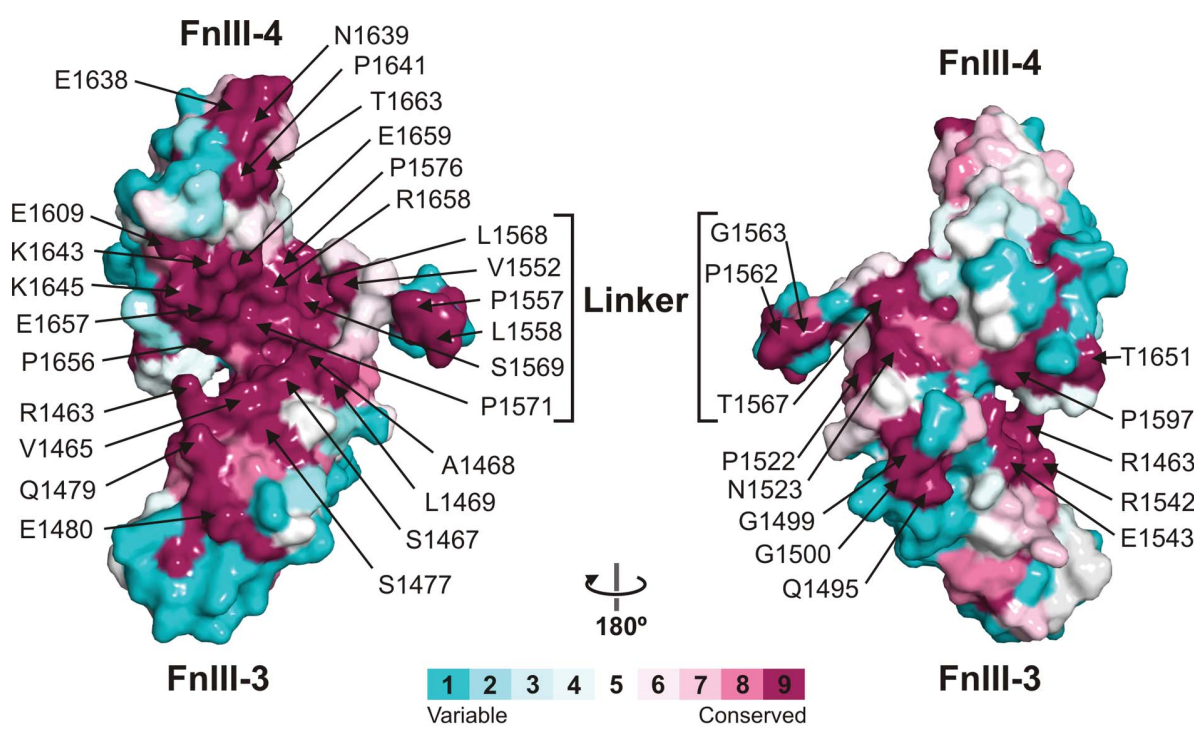

(a)

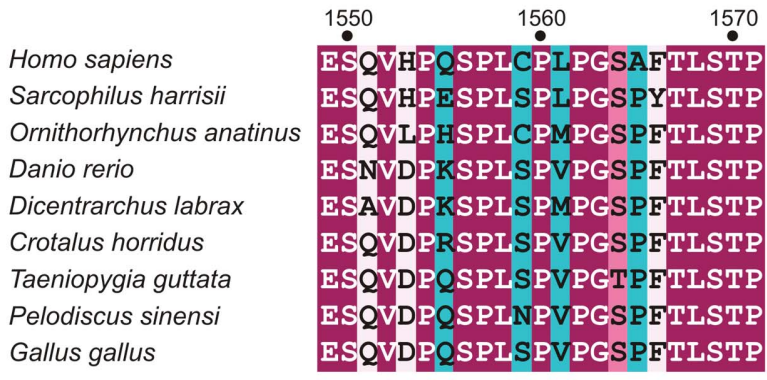

(b)

Figure 9

Conserved interdomain region in FnIII-3,4 of $\beta 4$. (a) Molecular surface of the FnIII-3,4 structure coloured by the evolutionary conservation of the amino acids in the $\beta 4$ sequences of 39 species calculated with the ConSurf server. The linker is shown in the conformation that yielded the best fit to the SAXS data. (b) Alignment of the sequences of the linker of nine species representative of the evolutionary variability. Positions are coloured according to the evolutionary conservation as in $(a)$. A complete alignment of the sequences of the $\beta 4$ FnIII-3,4 region of 39 species is shown in Supplementary Fig. S12. frequent conservation of functional sites in proteins prompted us to investigate whether the FnIII-3,4 structure contains evolutionary conserved patches that could correspond to potential interaction sites. Analysis of the sequences of integrin $\beta 4$ from mammals, birds, reptiles and fishes revealed an evolutionarily conserved surface spanning FnIII-3 and FnIII-4 at the inner side of the structure (Fig. 9). In addition, the final residues of the linker $\left({ }^{1567} \mathrm{TLSTP}^{1571}\right)$, which are also fully conserved, are predicted to lie between the two domains at the inner side. On the other hand, the outer surface is poorly conserved. In summary, the two FnIII domains and the linker form a continuous conserved surface that might correspond to an area of functional relevance.

\section{Discussion}

Large proteins with relatively long flexible linkers between rigid domains are often hard to crystallize. Solution structures of such proteins can be characterized by combining atomic resolution information on the local structure of the domains with lower-resolution information on global structure. Such approaches have been demonstrated by combining X-ray structures of the domains with DEER, SAXS and FRET data for the ESCRT-I (Boura et al., 2011) and ESCRT-II (Boura et al., 2012) complex and by combining NMR and DEER data to solve the interdomain arrangement in the Omp85 protein BamA (Ward et al., 2009) and the structure of the RsmE-RsmZ proteinRNA complex (Duss, Michel et al., 2014; Duss, Yulikov et al., 2014). By providing distance distributions rather than only mean distances, the DEER data allow the recognition of large-scale distributions of conformations, as is the case for ESCRT-I, ESCRT-II and RsmE-RsmZ, or to conclude that the relative domain orientation is well defined, as is the case for BamA and FnIII-3,4 of $\beta 4$. Although the X-ray structures of the individual domains and the DEER data on their own are sufficient to generate a model ensemble, we find that this ensemble is further constrained by the SAXS data with respect to both relative domain orientations and linker conformations. The approach introduced here allows quantitative estimation of the uncertainty of the structural model and is extensible to systems with multiple domains. Each added domain requires of the order of 12 additional DEER distribution constraints. The number of constraints required to specify a linker conformation depends on the linker 
length. At least two constraints per reference point between ten-residue segments of the linker are required to obtain a coarse ensemble. We expect that localization approaches, such as that demonstrated for lipoxygenase (Gaffney et al., 2012), will allow narrowing of the ensemble. Recent work on the proapoptotic protein Bax indicates that five DEER constraints per reference point provided good localization (Bleicken et al., 2014).

The compact structure of FnIII-3,4 revealed here has implications for understanding the organization and interactions of integrin $\beta 4$. The region of $\beta 4$ formed by the CS, FnIII-3,4 and the C-tail is believed to adopt a folded-back structure. Intramolecular FRET between cyan (CFP) and yellow (Venus) fluorescent proteins has been detected in cells expressing a $\beta 4$ construct that incorporates Venus right upstream of FnIII-3 and the CFP at the C-terminus (Frijns et al., 2012). In our structure, the N- and C-termini of FnIII-3,4 are $\sim 60-65 \AA$ apart, which is within the distance range that allows FRET between the CFP-Venus pair $(<100 \AA)$. In addition, since the CFP was at the end of the $\beta 4$ chain in this FRET sensor, the 86-residue C-tail might be projected towards the CS, contributing to the proximity of the CFP and Venus tags. We observed negligible interdomain conformational variability, which suggests that FnIII-3,4 does not behave as a flexible hinge that could bend in response to CS/ C-tail interactions. On the contrary, FnIII-3,4 probably acts as a rigid platform that promotes proximity of the CS and the C-tail.

FnIII-3,4 and the final portion of the CS (region 1436-1667) are sufficient for binding to BPAG1e (Koster et al., 2003). The last 21 residues of the CS do not alter the structure of FnIII3,4 , as observed by SAXS analysis of the 1436-1666 fragment (Manso \& de Pereda, unpublished results). Therefore, our structure is in a BPAG1e binding-active conformation of FnIII-3,4, which is likely to be the organization of this region in the complete $\alpha 6 \beta 4$ integrin. Interaction of $\beta 4$ with BPAG1e requires both FnIII-3 and FnIII-4, suggesting that the binding interface extends along the two domains. It is notable that the evolutionarily conserved surface in FnIII-3,4, which probably corresponds to a functional site, spreads throughout the two FnIII domains and the linker and could be involved in binding to BPAG1e. In fact, the clustering of conserved residues in FnIII- 3,4 is reminiscent of the plectin-binding site in $\beta 4$, which is the largest surface of FnIII-1,2 that is preserved among multiple species (Supplementary Fig. S13).

Three Tyr residues in FnIII-3,4 (Y1494, Y1526 and Y1642) have been implicated in phosphorylation-dependent signalling. Phospho-Y1494 is recognized by the SH2 domain of SHP2 (Bertotti et al., 2006), while the phosphotyrosinebinding (PTB) domain of Shc binds to phosphorylated Y1526 and possibly $\mathrm{Y} 1642$, which are within $\mathrm{N} X X \mathrm{pY}$ motifs (where $X$ is any amino acid; Dans et al., 2001). Phospho-Tyr ligands adopt extended conformations when bound to $\mathrm{SH} 2$ domains; similarly, residues upstream of $\mathrm{N} X X \mathrm{pY}$ motifs make $\beta$-sheet contacts with PTB domains (Kaneko et al., 2012). Therefore, the structural environments of Y1494, Y1526 and Y1642 are not compatible with binding to SH2 or PTB domains. This apparent contradiction can be reconciled if the FnIII fold is disrupted in the Tyr-phosphorylated form of $\beta 4$. For example, mechanical stretching of $\beta 4$ could expose cryptic Tyrphosphorylation sites. Alternatively, phosphorylation of Tyr could trigger unfolding of the FnIII domain or could prevent its refolding. The sulfate near Y1526 in the crystal structure suggests that FnIII-3 might tolerate phosphorylation at this position. Nonetheless, owing to the small exposure of Y1494 and Y1526 to the solvent, phosphates attached to these residues are likely to cause steric hindrance. The nearby acidic residues E1518, D1519 and E1501 could also create electrostatic repulsion with the phosphates. In addition, owing to the proximity between Y1494 and Y1526, simultaneous phosphorylation of these Tyr residues could destabilize FnIII-3 owing to repulsion between the phosphate groups, suggesting that multiple phosphorylation events might collaborate to fully trigger $\beta 4$-dependent signalling. It is also possible that phosphorylation of one residue might act as an initiator, relaxing the structure and favouring the phosphorylation of the companion Tyr in a sequential manner. Interestingly, Y1494 has been proposed as a main regulator of $\alpha 6 \beta 4$ signalling in cancer cells (Dutta \& Shaw, 2008). In contrast to Y1494 and Y1526 in FnIII-3, Y1642 in FnIII-4 sits in a wider pocket surrounded by uncharged residues. Therefore, phosphorylation of Y1642 is less likely to destabilize FnIII-4 and correlates with a minor role of Y1642 in the recruitment of Shc (Dans et al., 2001).

In summary, FnIII-3,4 emerges as a structural and functional unit within the $\beta 4$ integrin. Global changes in the interdomain arrangement, for example in response to pulling forces, could alter or unveil binding sites in FnIII-3,4, suggesting that $\alpha 6 \beta 4$ might act as a mechanosensor. Conversely, local changes in one of these FnIII domains could propagate to other regions of $\beta 4$, such as the CS and C-tail. This work provides a detailed structural framework to better investigate the role $\beta 4$ in epithelial homeostasis and in carcinoma progression.

\section{Related literature}

The following references are cited in the Supporting Information for this article: de Pereda, Lillo et al. (2009), Robert \& Gouet (2014) and Sievers et al. (2011).

\section{Acknowledgements}

We acknowledge the Swiss Light Source and the EMBL for the provision of synchrotron-radiation facilities. This work was supported by the Spanish Ministry of Economy and Competitiveness and the European Regional Development Fund grants BFU2009-08389 and BFU2012-32847 (to JMdP), by SNF grant 200020_14441 (to GJ) and by the Netherlands Science Foundation and the Dutch Cancer Society (to AS). NAG received a JAE contract (CSIC), RMB received Juan de la Cierva and Ramon y Cajal contracts and JAM received a fellowship from the University of Salamanca. This research received funding from the European Community's Seventh 
Framework Programme (FP7/2007-2013) under BioStruct-X (grant agreement No. 283570).

\section{References}

Abrahams, J. P. \& Leslie, A. G. W. (1996). Acta Cryst. D52, 30-42.

Afonine, P. V., Grosse-Kunstleve, R. W., Echols, N., Headd, J. J., Moriarty, N. W., Mustyakimov, M., Terwilliger, T. C., Urzhumtsev, A., Zwart, P. H. \& Adams, P. D. (2012). Acta Cryst. D68, 352-367. Alonso-García, N., Inglés-Prieto, A., Sonnenberg, A. \& de Pereda, J. M. (2009). Acta Cryst. D65, 858-871.

Ashkenazy, H., Erez, E., Martz, E., Pupko, T. \& Ben-Tal, N. (2010). Nucleic Acids Res. 38, W529-W533.

Baker, N. A., Sept, D., Joseph, S., Holst, M. J. \& McCammon, J. A. (2001). Proc. Natl Acad. Sci. USA, 98, 10037-10041.

Barczyk, M., Carracedo, S. \& Gullberg, D. (2010). Cell Tissue Res. 339, 269-280.

Bernadó, P., Mylonas, E., Petoukhov, M. V., Blackledge, M. \& Svergun, D. I. (2007). J. Am. Chem. Soc. 129, 5656-5664.

Bertotti, A., Comoglio, P. M. \& Trusolino, L. (2006). J. Cell Biol. 175, 993-1003.

Bleicken, S., Jeschke, G., Stegmueller, C., Salvador-Gallego, R., García-Sáez, A. J. \& Bordignon, E. (2014). Mol. Cell, 56, 496-505.

Boura, E., Różycki, B., Chung, H. S., Herrick, D. Z., Canagarajah, B., Cafiso, D. S., Eaton, W. A., Hummer, G. \& Hurley, J. H. (2012). Structure, 20, 874-886.

Boura, E., Rózycki, B., Herrick, D. Z., Chung, H. S., Vecer, J., Eaton, W. A., Cafiso, D. S., Hummer, G. \& Hurley, J. H. (2011). Proc. Natl Acad. Sci. USA, 108, 9437-9442.

Campbell, I. D. \& Humphries, M. J. (2011). Cold Spring Harb. Perspect. Biol. 3, a004994.

Chacón, P., Díaz, J. F., Morán, F. \& Andreu, J. M. (2000). J. Mol. Biol. 299, 1289-1302.

Chen, V. B., Arendall, W. B., Headd, J. J., Keedy, D. A., Immormino, R. M., Kapral, G. J., Murray, L. W., Richardson, J. S. \& Richardson, D. C. (2010). Acta Cryst. D66, 12-21.

Chung, H. J. \& Uitto, J. (2010). Dermatol. Clin. 28, 43-54.

Dans, M., Gagnoux-Palacios, L., Blaikie, P., Klein, S., Mariotti, A. \& Giancotti, F. G. (2001). J. Biol. Chem. 276, 1494-1502.

Diederichs, K. \& Karplus, P. A. (1997). Nature Struct. Mol. Biol. 4, 269-275.

Durand, D., Vivès, C., Cannella, D., Pérez, J., Pebay-Peyroula, E., Vachette, P. \& Fieschi, F. (2010). J. Struct. Biol. 169, 45-53.

Duss, O., Michel, E., Yulikov, M., Schubert, M., Jeschke, G. \& Allain, F. H. (2014). Nature (London), 509, 588-592.

Duss, O., Yulikov, M., Jeschke, G. \& Allain, F. H. (2014). Nature Commun. 5, 3669.

Dutta, U. \& Shaw, L. M. (2008). Cancer Res. 68, 8779-8787.

Emsley, P., Lohkamp, B., Scott, W. G. \& Cowtan, K. (2010). Acta Cryst. D66, 486-501.

Engh, R. A. \& Huber, R. (1991). Acta Cryst. A47, 392-400.

Franke, D. \& Svergun, D. I. (2009). J. Appl. Cryst. 42, 342-346.

Frijns, E., Kuikman, I., Litjens, S., Raspe, M., Jalink, K., Ports, M., Wilhelmsen, K. \& Sonnenberg, A. (2012). Mol. Biol. Cell, 23, 14751485.

Gaffney, B. J., Bradshaw, M. D., Frausto, S. D., Wu, F., Freed, J. H. \& Borbat, P. (2012). Biophys. J. 103, 2134-2144.

García-Alvarez, B., Bobkov, A., Sonnenberg, A. \& de Pereda, J. M. (2003). Structure, 11, 615-625.

Geerts, D., Fontao, L., Nievers, M. G., Schaapveld, R. Q., Purkis, P. E., Wheeler, G. N., Lane, E. B., Leigh, I. M. \& Sonnenberg, A. (1999). J. Cell Biol. 147, 417-434.

Gromov, I., Shane, J., Forrer, J., Rakhmatoullin, R., Rozentzwaig, Y. \& Schweiger, A. (2001). J. Magn. Reson. 149, 196-203.

Hagens, T. von, Polyhach, Y., Sajid, M., Godt, A. \& Jeschke, G. (2013). Phys. Chem. Chem. Phys. 15, 5854.
Hovmöller, S., Zhou, T. \& Ohlson, T. (2002). Acta Cryst. D58, 768-776.

Hynes, R. O. (2002). Cell, 110, 673-687.

Jeschke, G. (2012). Annu. Rev. Phys. Chem. 63, 419-446.

Jeschke, G., Chechik, V., Ionita, P., Godt, A., Zimmermann, H., Banham, J., Timmel, C. R., Hilger, D. \& Jung, H. (2006). Appl. Magn. Reson. 30, 473-498.

Kabsch, W. (1976). Acta Cryst. A32, 922-923.

Kabsch, W. (2010). Acta Cryst. D66, 125-132.

Kaneko, T., Joshi, R., Feller, S. M. \& Li, S. S. C. (2012). Cell Commun. Signal. 10, 32.

Kleywegt, G. J. (1997). J. Mol. Biol. 273, 371-376.

Konarev, P. V., Volkov, V. V., Sokolova, A. V., Koch, M. H. J. \& Svergun, D. I. (2003). J. Appl. Cryst. 36, 1277-1282.

Koster, J., Geerts, D., Favre, B., Borradori, L. \& Sonnenberg, A. (2003). J. Cell Sci. 116, 387-399.

Koster, J., van Wilpe, S., Kuikman, I., Litjens, S. H. \& Sonnenberg, A. (2004). Mol. Biol. Cell, 15, 1211-1223.

Kozin, M. B. \& Svergun, D. I. (2001). J. Appl. Cryst. 34, 33-41.

Krivov, G. G., Shapovalov, M. V. \& Dunbrack, R. L. Jr (2009). Proteins, 77, 778-795.

Langer, G., Cohen, S. X., Lamzin, V. S. \& Perrakis, A. (2008). Nature Protoc. 3, 1171-1179.

Margadant, C., Frijns, E., Wilhelmsen, K. \& Sonnenberg, A. (2008). Curr. Opin. Cell Biol. 20, 589-596.

McCoy, A. J., Grosse-Kunstleve, R. W., Adams, P. D., Winn, M. D., Storoni, L. C. \& Read, R. J. (2007). J. Appl. Cryst. 40, 658-674.

Murgia, C., Blaikie, P., Kim, N., Dans, M., Petrie, H. T. \& Giancotti, F. G. (1998). EMBO J. 17, 3940-3951.

Ortega, A., Amorós, D. \& García de la Torre, J. (2011). Biophys. J. 101, 892-898.

Orthaber, D., Bergmann, A. \& Glatter, O. (2000). J. Appl. Cryst. 33, 218-225.

Pape, T. \& Schneider, T. R. (2004). J. Appl. Cryst. 37, 843-844.

de Pereda, J. M., Lillo, M. P. \& Sonnenberg, A. (2009). EMBO J. 28, 1180-1190.

de Pereda, J. M., Ortega, E., Alonso-Garcia, N., Gómez-Hernández, M. \& Sonnenberg, A. (2009). Cell Adh. Migr. 3, 361-364.

de Pereda, J. M., Wiche, G. \& Liddington, R. C. (1999). EMBO J. 18, 4087-4095.

Petoukhov, M. V., Franke, D., Shkumatov, A. V., Tria, G., Kikhney, A. G., Gajda, M., Gorba, C., Mertens, H. D. T., Konarev, P. V. \& Svergun, D. I. (2012). J. Appl. Cryst. 45, 342-350.

Polyhach, Y., Bordignon, E. \& Jeschke, G. (2011). Phys. Chem. Chem. Phys. 13, 2356.

Rezniczek, G. A., de Pereda, J. M., Reipert, S. \& Wiche, G. (1998). J. Cell Biol. 141, 209-225.

Richter, S., Wenzel, A., Stein, M., Gabdoulline, R. R. \& Wade, R. C. (2008). Nucleic Acids Res. 36, W276-W280.

Robert, X. \& Gouet, P. (2014). Nucleic Acids Res. 42, W320-W324.

Shaw, L. M. (2001). Mol. Cell. Biol. 21, 5082-5093.

Sheldrick, G. M. (2010). Acta Cryst. D66, 479-485.

Shimanouchi, T. \& Mizushima, S. (1955). J. Chem. Phys. 23, 707-711.

Sievers, F., Wilm, A., Dineen, D., Gibson, T. J., Karplus, K., Li, W., Lopez, R., McWilliam, H., Remmert, M., Söding, J., Thompson, J. D. \& Higgins, D. G. (2011). Mol. Syst. Biol. 7, 539.

Sitkoff, D., Sharp, K. A. \& Honig, B. (1994). J. Phys. Chem. 98, 19781988.

Sugeta, H. \& Miyazawa, T. (1967). Biopolymers, 5, 673-679.

Svergun, D., Barberato, C. \& Koch, M. H. J. (1995). J. Appl. Cryst. 28, 768-773.

Theobald, D. L. \& Steindel, P. A. (2012). Bioinformatics, 28, 19721979.

Tschaggelar, R., Kasumaj, B., Santangelo, M. G., Forrer, J., Leger, P., Dube, H., Diederich, F., Harmer, J., Schuhmann, R., García-Rubio, I. \& Jeschke, G. (2009). J. Magn. Reson. 200, 81-87.

Valentini, E., Kikhney, A. G., Previtali, G., Jeffries, C. M. \& Svergun, D. I. (2014). Nucleic Acids Res. 43, D357-D363. 


\section{research papers}

Volkov, V. V. \& Svergun, D. I. (2003). J. Appl. Cryst. 36, 860864.

Vonrhein, C., Blanc, E., Roversi, P. \& Bricogne, G. (2007). Methods Mol. Biol. 364, 215-230.

Ward, R., Zoltner, M., Beer, L., El Mkami, H., Henderson, I. R.,
Palmer, T. \& Norman, D. G. (2009). Structure, 17, 1187-1179.

Wilhelmsen, K., Litjens, S. H. \& Sonnenberg, A. (2006). Mol. Cell. Biol. 26, 2877-2886.

Winn, M. D. et al. (2011). Acta Cryst. D67, 235-242.

Wriggers, W. (2010). Biophys. Rev. 2, 21-27. 